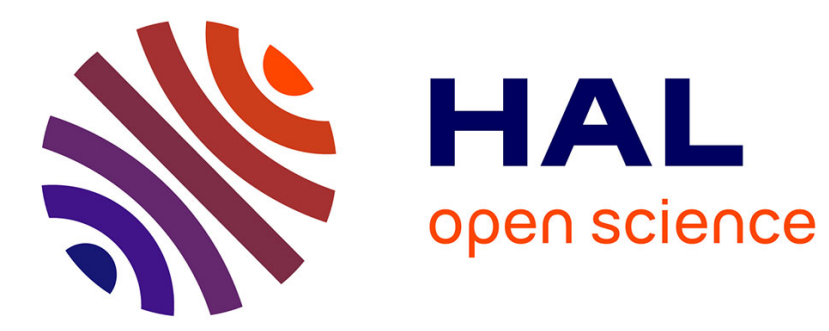

\title{
Shadowgraph, Schlieren and interferometry in a 2D cavitating channel flow
}

Cyril Mauger, Loïc Méès, Marc Michard, Alexandre Azouzi, Stéphane Valette

\section{To cite this version:}

Cyril Mauger, Loïc Méès, Marc Michard, Alexandre Azouzi, Stéphane Valette. Shadowgraph, Schlieren and interferometry in a 2D cavitating channel flow. Experiments in Fluids, 2012, 53 (6), pp.1895-1913. 10.1007/s00348-012-1404-3 . hal-00780132

\section{HAL Id: hal-00780132 https://hal.science/hal-00780132}

Submitted on 4 Feb 2016

HAL is a multi-disciplinary open access archive for the deposit and dissemination of scientific research documents, whether they are published or not. The documents may come from teaching and research institutions in France or abroad, or from public or private research centers.
L'archive ouverte pluridisciplinaire HAL, est destinée au dépôt et à la diffusion de documents scientifiques de niveau recherche, publiés ou non, émanant des établissements d'enseignement et de recherche français ou étrangers, des laboratoires publics ou privés. 


\title{
Shadowgraph, Schlieren and Interferometry in a 2D Cavitating Channel Flow
}

\author{
Cyril MAUGER ${ }^{\text {a }}$, Loïc MÉÈS ${ }^{\text {a,* }}$, Marc MICHARD ${ }^{\text {a }}$, Alexandre AZOUZI ${ }^{\text {a }}$ and \\ Stéphane VALETTE ${ }^{\mathrm{b}}$ \\ ${ }^{a}$ Laboratoire de Mécanique des Fluides et d'Acoustique (LMFA), CNRS \\ UMR5509 - Ecole Centrale de Lyon - INSA de Lyon - Université Claude \\ Bernard - Lyon 1, Ecully \\ ${ }^{b}$ Laboratoire de Tribologie et Dynamique des Systèmes (LTDS), CNRS UMR5513 \\ - Ecole Centrale de Lyon - Ecole Nationale d'Ingénieurs de Saint Etienne \\ *Corresponding Author loic.mees@ec-lyon.fr
}

\begin{abstract}
Cavitation plays an important role in fuel atomization mechanisms but the physics of cavitation and its impact on spray formation and injector efficiency are not well documented yet. Experimental investigations are required to support the development and the validation of numerical models and the design of tomorrow's injectors, in the context of pollutant and fuel consumption reduction. The complexity of modern injectors and the extreme conditions of injection do not facilitate experimental investigations. In this paper, experiments are conducted in a simplified geometry. The model nozzle consists of a transparent 2D micro-channel supplied with a test-oil (ISO 4113). Three different optical techniques are proposed to investigate the channel flow, with the pressure drop between upstream and downstream chambers as a parameter. A shadowgraph-like imaging technique allows the observation of cavitation inception and vapor cavities development throughout the channel. The technique also reveals the presence of density gradients (pressure or temperature) in the channel flow. However, this additional information is balanced by difficulties in image interpretation, which are discussed in the paper. In addition, a combination of Schlieren technique and interferometric imaging is used to measure the density fields inside the channel. The three techniques results are carefully analysed and confronted. These results reveal a wealth of information on the flow, with pressure waves generated by bubble collapses, turbulence in the wake of vapor cavities and bubble survival in flow regions of high pressure. Our results also show that cavitation inception is located in the shear layers between the recirculation zones and the main flow, relatively far from the inlet corner, where the pressure is minimum in average. To explain this behavior, we propose a scenario of cavitation inception based on the occurrence and the growing of instabilities in the shear layers.
\end{abstract}

Keywords: Direct injection / Cavitation / Channel flow / Shadowgraph / Interferometry / Schlieren / Nozzle 


\section{Introduction}

In the modern internal combustion engine, diesel or gasoline is directly injected into the combustion chamber. The liquid fuel is injected through a hole-type nozzle with very high injection pressure, to produce a spray of fine droplets. The spray characteristics are crucial, since they determine the evaporation and mixing processes before combustion. In the nozzle holes, the flow is strongly accelerated and the pressure may drop down to the fuel vapor pressure, leading to cavitation. Cavitation is generally regarded as a source of problems: noise, vibrations, efficiency loss and damage. In the case of fuel injection in particular, it leads to a limitation of the flow rate. However, cavitation offers some advantages by preventing nozzles from fouling and possibly enhancing atomization processes.

Cavitation in a diesel nozzle has been studied in the early work of Bergwerk (1959). In this paper, vapor cavities have been observed in transparent and upscaled nozzles. In (Nurick 1976) the development of cavitation has been observed in sharp-edged orifices. Nurick proposed a one-dimensional model to describe cavitation inception and development as a function of two dimensionless parameters (the cavitation number and the discharge coefficient). In the same paper, the great influence of the orifice entrance geometry on cavitation is pointed out. The influence of geometric parameters, as the entrance radius (Soteriou et al. 1995; Schmidt et al. 1997; Winklhofer et al. 2001) or the orifice taper (Winklhofer et al. 2003; Payri et al. 2005) have been later confirmed experimentally and numerically. An effect of roughness at orifice wall and orifice inlet has been also considered (Jung et al. 2008; Chang et al. 2006; Winklhofer et al. 2003). These studies have shown that very weak variations in the orifice geometry could have a disproportionate influence on cavitation. A perfect control of the geometry and surface conditions is then suitable to study nozzle cavitation experimentally, especially when comparisons with Computational Fluid Dynamics are concerned.

Experimental study of nozzle cavitation in real conditions is difficult. Direct observation of such a small and fast two phase flow requires high resolutions in both space and time. The nozzles must be transparent and resist to high injection pressure and chocks caused by the needle lift. As a consequence, only a few experimental studies have been done in realistic conditions (Badock, 1999) while 
most of them have been conducted on simplified geometries: up-scaled nozzles, lower pressure and velocities... In the present paper, cavitation is studied in a quasi-two dimensional orifice (channel), in permanent flow condition (without needle lift) and lower pressure levels than those currently used in real injectors. Such a simplification has been already adopted in previous works (Roosen 2007; Winklhofer et al. 2001). It provides several advantages in terms of optical access and image interpretation, notably. In this paper, we also take advantage of the $2 \mathrm{D}$ geometry to control and characterize the channel geometry and surface roughness. Experimental results obtained in this simplified geometry, with simplified working conditions, cannot be directly transferred to real diesel injectors. However, these simplifications allow to obtain high quality images of the flow and quantitative information to be used as a reference for Computational Fluid Dynamics (CFD) results and the cavitation models involved. In addition, such a simplified experimental set-up is suitable to study the physical mechanisms involved (cavitation inception and development, bubble collapse, ...) to enhance our general understanding of nozzle cavitation. The channel flow is visualized as a function of the pressure drop, by using a shadowgraph-like technique, the Schlieren method and interferometric imaging. We pay a special attention to images interpretation and to the inception of cavitation, by confronting results obtained with the three different techniques.

The paper is organized as follows. In Section 2, the apparatus set-up used to generate and control the channel flow is presented. Section 3 provides the general features of cavitation in channel flows. In Section 4, the principles of shadowgraph is recalled and the shadowgraph-like optical arrangement proposed in this paper is presented. Images of the flow obtained with this arrangement are then discussed, as a function of the pressure drop. A particular attention is paid to cavitation inception and to qualitative information on density gradients, provided by shadowgraph effect. Section 5 is dedicated to the Schlieren method. The basic features of the technique are recalled and density profiles are deduced from the Schlieren images by integration. In section 6, an interferometric imaging arrangement is presented. Unlike the previous technique, it provides quantitative information on the channel flow density. Density fields are deduced from the interferometric images by taking advantage of qualitative information provided by the Schlieren method. Section 7 is a discussion based on the three techniques 
results. Density fields, density gradients and shadowgraph-like images are discussed together to further detail the shadowgraph-like images interpretation, to discuss the mechanism of cavitation inception, and to study the bubble detachments and the pressure waves appearing in these images.

\section{Experimental set-up}

The channel consists of two separated metal sheets, sandwiched between a pair of glass windows (Fig. 1). The channel is continuously supplied with fuel (test oil SHELL V-Oel-1404, ISO 4113) by a volumetric pump, through holes directly drilled into the glass windows. A by-pass and valves allow one to set the flow rate in the channel, as well as upstream and downstream pressures. The temperature is regulated by using a heat exchanger and the fuel is filtered before returning in a tank at atmospheric pressure. A flow meter temperature and pressure sensors are used to measure the flow conditions.

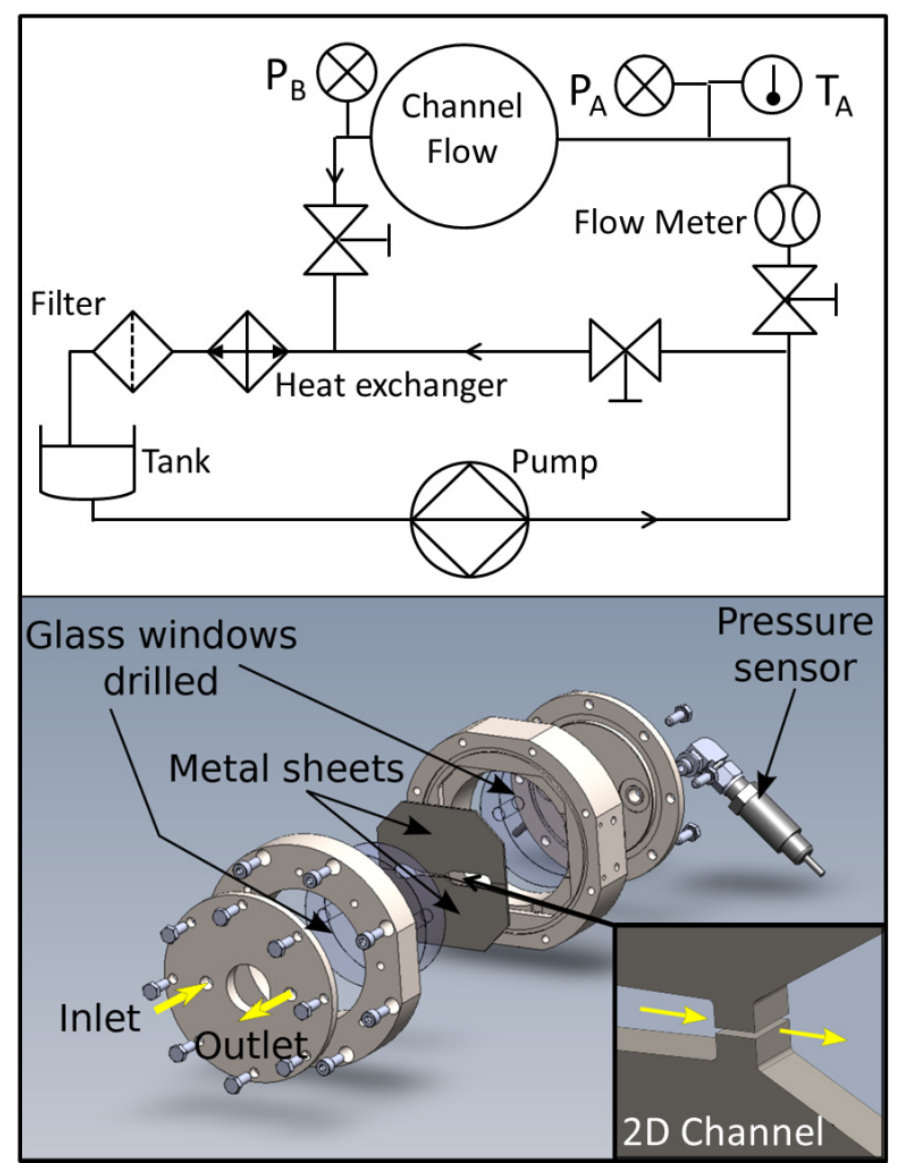

Figure 1: Experimental set-up. 
The use of separated metal sheets allows a better control of the channel geometry and surface roughness. Fig. 2 shows Scanning Electron Microscope (SEM) images of the channel. Fig. 2a shows the whole channel The inserts (Fig. $2 b$ to fig.2e) focus on the channel entrance with tilted and non-tilted views of the lower and upper entrance corners). The channel is about $400 \mu \mathrm{m}$ height (exact dimensions are reported in Fig. 2a) and $1475 \mu \mathrm{m}$ long, with a weak taper of about $0.4^{\circ}$. The channel depth is $L=2 \mathrm{~mm}$. The surfaces constituting the channel walls have been mirror-polished. Surface roughness has been characterized with an optical profilometer, by an arithmetic mean roughness $R a<0.1 \mu \mathrm{m}$. At the channel entrance corners, the rounding is evaluated to be less than $10 \mu \mathrm{m}$.

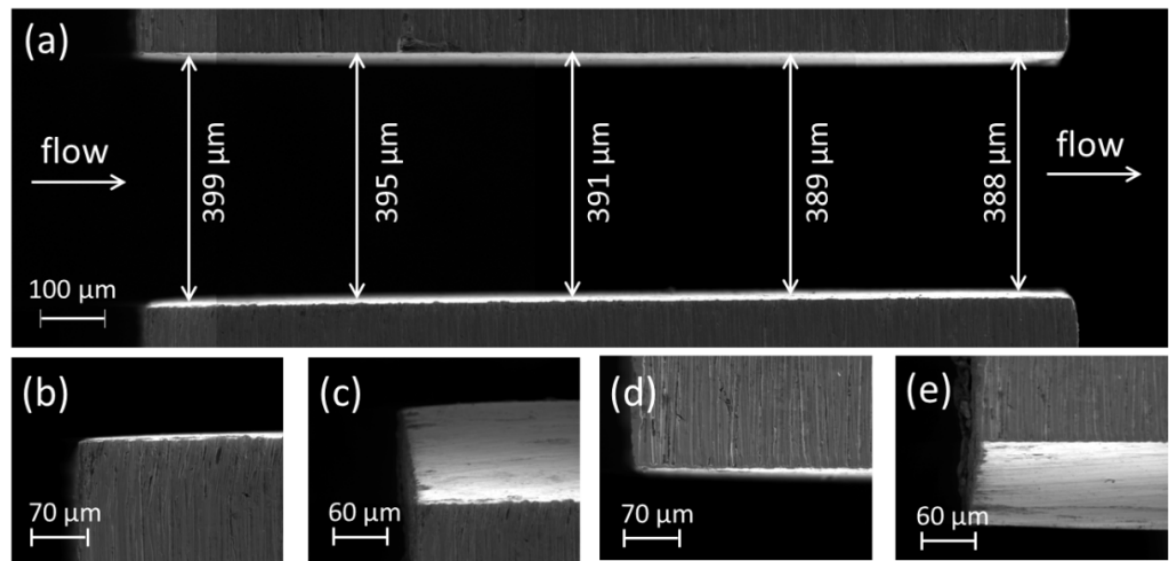

Figure 2: SEM images of the channel. a: overview of the channel ; b: lower entrance corner (nontilted view) ; c: lower entrance corner (tilted view) ; d : upper entrance corner (non-tilted view); e : upper entrance corner (tilted view).

Three optical techniques have been used to visualize the channel flow: a shadowgraph-like technique, the Schlieren method and an interferometric imaging technique. The corresponding setups will be introduced later in the paper. The following section is dedicated to the presentation of the general features of the channel flow.

\section{General features}

Fig. 3 is a schematic of the channel flow. The fluid flows between A and B under the effect of the pressure drop $\Delta P=P_{A}-P_{B}$. In the following, the upstream pressure $P_{A}$ will remain constant, while pressure drop and flow rate will be increased by decreasing the downstream pressure $P_{B}$. At the orifice inlet, the 
strong change in cross section and flow direction induces a separation of the boundary layers from the orifice walls and the formation of a vena contracta. Recirculation zones appear between the walls and the main flow which undergoes a supplementary contraction in the central part of the channel.

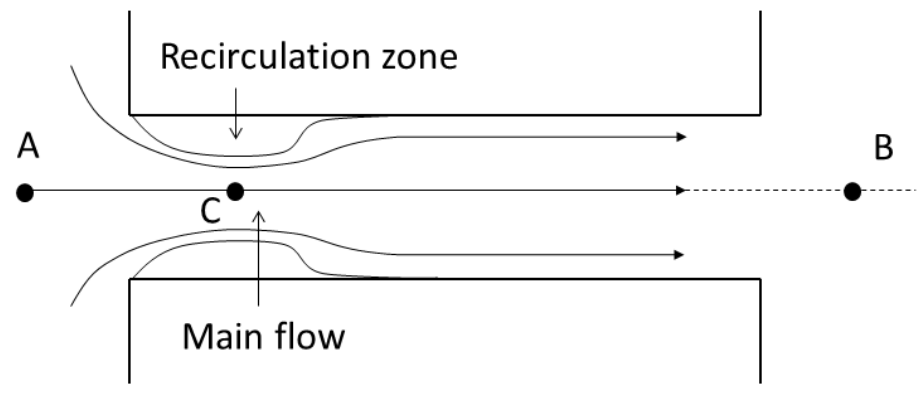

Figure 3: Schematic of the orifice flow.

A one-dimensional model is proposed by Nurick (1976) to account for the cavitation in nozzle flow. This simple model is based on two dimensionless parameters: the discharge coefficient and the cavitation number. The discharge coefficient is defined as the ratio between the actual mass flow rate $\dot{m}$ and the theoretical mass flow rate, given the pressure drop $\Delta P$ and the orifice section $S_{0}$. More precisely,

$$
C_{D}=\frac{\dot{m}}{S_{0} \sqrt{2 \rho\left(P_{A}-P_{B}\right)}}
$$

where $\rho$ is the fluid density.

The cavitation number is defined by Nurick (1976) as the ratio

$$
K=\frac{P_{A}-P_{v}}{P_{A}-P_{B}}
$$

where $P_{v}$ is the vapor pressure of the fluid. $K$ decreases with decreasing $P_{B}$ (increasing $\Delta P$ ) and cavitation occurs at low value of $K$ (typically lower than 2). Starting with low pressure drop $\Delta P$, the mass flow rate increases when $P_{B}$ decreases and the pressure $P_{C}$, at the minimum section of the contracted flow, decreases. Following Nurick model, cavitation incepts in the orifice when $P_{C}$ 
reaches the vapor pressure. The flow rate then reaches a maximum value and remains constant for further decreasing of $P_{B}$ :

$$
\dot{m}_{c r i t}=S_{C} \sqrt{2 \rho\left(P_{A}-P_{v}\right)}
$$

where $S_{C}$ is the contracted flow section in C. Under this choked flow condition $\left(K<K_{c r i t}\right)$, the discharge coefficient can be related to the contraction coefficient:

$$
C_{C}=S_{C} / S_{0}
$$

by

$$
C_{D}=C_{C} \sqrt{K}
$$

Still following Nurick (1976): for $K<K_{c r i t}$, the flow is two-phase, the flow rate is chocked and the discharge coefficient is proportional to the square root of cavitation number; for $K>K_{c r i t}$, the flow remains single-phase and the discharge coefficient is almost constant at about 0.8 .

Fig. 4 displays the mass flow rate measured in the channel as a function of the square root of pressure drop. Pressure drop is changed by changing downstream pressure $P_{B}$ at constant upstream pressure $P_{A}=50$ bar. The flow temperature is maintained to $32^{\circ} \mathrm{C}$. At this temperature and at a pressure of $50 \mathrm{bar}$, the fluid density is $\rho=815.4 \mathrm{~kg} \cdot \mathrm{m}^{-3}$. The curve evolution is simple and can be described in two parts. At low pressure drop, the mass flow rate is almost proportional to the pressure drop square root. This reflects that the discharge coefficient $C_{D}$ is almost constant in these conditions. We deduce from the slope of the curve (with $\mathrm{S}_{0}=7.74$ $\left.10^{-7} \mathrm{~m}^{2}\right)$ that $C_{D}=0.795$ for $\sqrt{\Delta P}<5.8\left(P_{B}>16.1\right.$ bar, $\left.K>1.47\right)$. For greater pressure drop, the mass flow rate remains constant. This reflects the linear relation between $C_{D}$ and $K^{1 / 2}$ of Eq. (5), with a contraction coefficient $C_{C}=0.647$ deduced from Eqs. (3) and (4). 


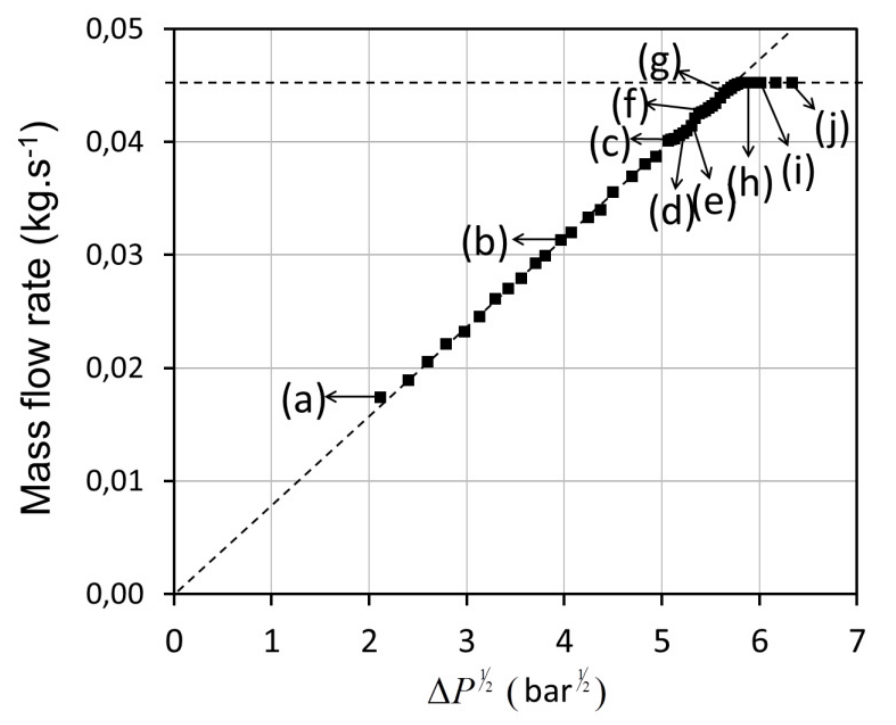

Figure 4: Mass flow rate in the channel as a function of the square root of pressure drop. The letters (a) to (j) refer to Fig. 9, where images recorded in the same pressure drop conditions are presented.

\section{Shadowgraph-like imaging}

Almost all the observations of cavitating flows in nozzles are based on shadowgraph or backlight imaging configuration. Strictly speaking, shadowgraph is a density sensitive technique, based on a back illumination and an appropriate defocusing. By extension, the term shadowgraph is often misused to describe more general types of backlight images. In this paper, backlit images that are sensitive to density gradient are presented, but the set-up differs somewhat from the standard shadowgraph, as described by Merzkirch (1974) for example.

\section{The principle of the standard shadowgraph technique}

Before introducing our optical arrangement in details, let us recall the main feature of the shadowgraph technique. Fig. 5 is a schematic of shadowgraph arrangement, strictly speaking. A point light source is collimated to illuminate the disturbed medium $D$. Images are recorded on a CCD sensor (or a photographic plate) by using an imaging lens which is defocused with respect to the disturbed medium. More precisely, the imaging lens is focused onto a plane $O$, distant by $l$ from the disturbed medium $D$. In the presence of refractive index gradients (related to density gradients) in $D$, an individual light ray undergoes a curved trajectory, leading to a displacement $\delta x$ and a deviation angle $\varepsilon_{x}$ with respect to 
the undisturbed ray at the medium $D$ exit. Assuming that rays undergo only infinitesimal deviations (i.e. $x$ and $y$ coordinates of the ray are the same at the medium entrance and exit) but have non-negligible curvature, the deflection angle is written as:

$$
\mathcal{E}_{x} \approx \tan \varepsilon_{x} \approx \int_{s_{0}}^{s_{1}} \frac{1}{n} \frac{\partial n}{\partial x} d s
$$

where integration is done along the ray trajectory through $D$.

In plane $O$, the ray displacement $\Delta x$ is roughly proportional to $l$ and to angle $\varepsilon_{x}$. Considering all the rays which contribute to image formation, displacements $\Delta x$ (and equivalent displacements $\Delta y$ along $y$ direction) lead to a redistribution of light intensity in plane $O$ (and consequently in image plane) which depends on the refractive index $n$ variations in the disturbed medium. Under the assumption of infinitesimal deviation previously introduced, the relative change in recorded intensity with respect to the undisturbed intensity can be approximated by

$$
\frac{\Delta I}{I} \approx-l \int_{s_{0}}^{s_{1}}\left(\frac{\partial^{2}}{\partial^{2} x}+\frac{\partial^{2}}{\partial^{2} y}\right)(\ln n) d s
$$

Assuming a 2D flow and still assuming infinitesimal ray displacements in $D$, Eq. (7) becomes

$$
\frac{\Delta I(x, y)}{I(x, y)} \approx-l L\left(\frac{\partial^{2}}{\partial^{2} x}+\frac{\partial^{2}}{\partial^{2} y}\right)(\ln n(x, y))
$$

Shadowgraph intensity distribution is then roughly proportional to the second derivative of the refractive index, to the depth $L$ of the disturbed medium and to the distance $l$. In principle $l$ can be adjusted to enhance or reduce the sensitivity of the technique to small density variations.

In principle, Eq. (8) can be integrated to obtain the refractive index (or the density) distribution in the medium. In practice, shadowgraph only provides qualitative information on flow density. Firstly, the technique is highly sensitive to any source of noise, as a double integration is required to obtain the density field. Secondly, the calibration of the technique appears to be a very difficult task as samples with a well-known second derivative of the refractive index must be used. 


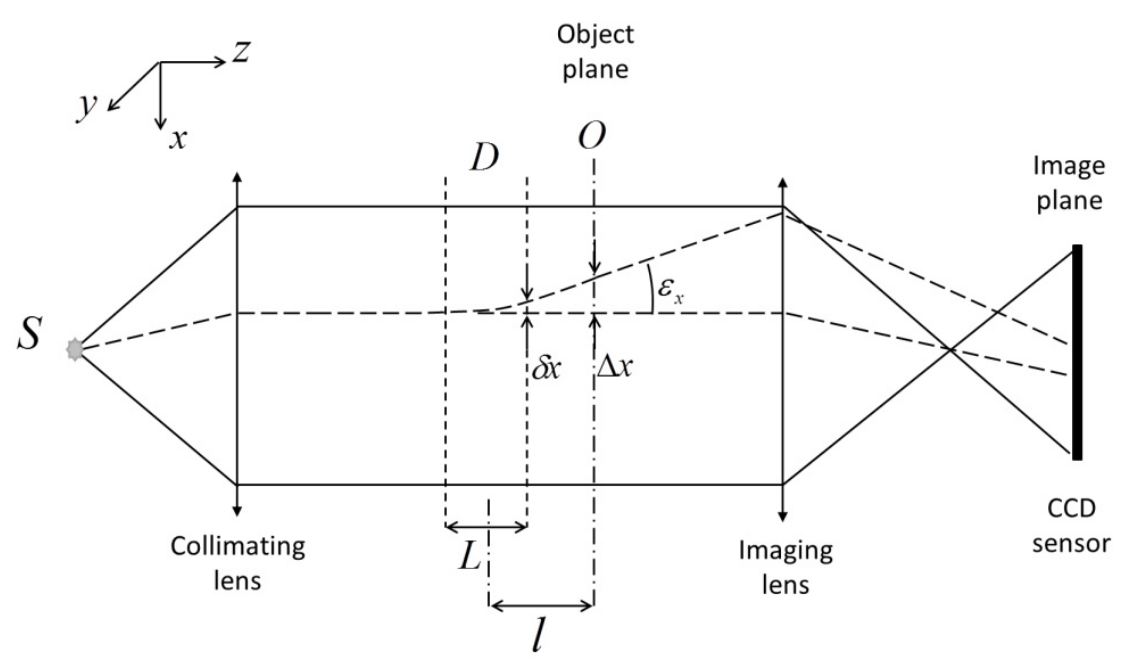

Figure 5: Standard shadowgraph arrangement.

\section{Shadowgraph-like arrangement}

In the shadowgraph-like configuration considered in this paper, the light source and the camera are located on opposite sides of the channel (Fig. 6). However, it differs from the standard shadowgraph arrangement described above as the camera lens is focused onto the channel flow, in order to visualize bubbles and vapor cavity as usually done in backlit imaging. Backlit imaging is based on ray deflection by interfaces. Assuming that interfaces are never perfectly flat and oriented perpendicularly to the incident rays, most of rays crossing an interface are deviated with an angle greater than the collection angle, whatever they are reflected or refracted through the interface. As a consequence, bubbles and vapor cavities appear dark in the image. On the contrary, liquid regions would appear bright, as (in absence of density gradient) they do not induce any ray deviation. In the present case however, density gradients and the channel depth $L$ are large enough to produce visible shadowgraph effect, as shown later in this section (Fig. 8 and 9).

The shadowgraph-like arrangement can be viewed as a backlit imaging arrangement which is sensitive to density gradient. Due to the very small size of the channel, the use of a large optical magnification is required. With flow velocities up to $70 \mathrm{~m} \cdot \mathrm{s}^{-1}$, an extremely short light pulse is needed to optically freeze the flow. In addition, an incoherent light source is suitable to avoid speckle on the images. To fulfill these requirements, a light source is generated by focusing the second harmonic of a Nd:YAG pulsed laser (wavelength $\lambda_{\mathrm{YAG}}=532$ 
$\mathrm{nm}$ and pulse duration of $6 \mathrm{~ns}$ ) on a fluorescing polymethyl-methacrylate (PMMA) sheet. The fluorescence emission is collimated and the remaining laser light is filtered by using a band reject filter (Notch filter $\lambda_{N}=533 \pm 8 \mathrm{~nm}$ ). A dual head laser is used to produce time delayed light pulses with $12 \mathrm{~ns}$ duration (FWHM) and a broad spectrum (about $100 \mathrm{~nm}$ ) centered on $\lambda_{\mathrm{F}}=592 \mathrm{~nm}$ (Fig. 7). The time delay between the two pulses is adjustable, down to about $30 \mathrm{~ns}$. Images are recorded by means of an optical zoom (OPTEM 125C), at low repetition rate (1Hz), on a $2048 \times 2048$ pixels, 10-bit CCD camera. The resulting resolution is $1.15 \mu \mathrm{m} / \mathrm{px}$. Using the double-frame mode of the camera and the dual pulse system, couples of images separated by $285 \mathrm{~ns}$ have been recorded. The arrangement then allows the tracking of rapid events, as bubble collapses (section 7) or the production of velocity information, which is not detailed in this paper.

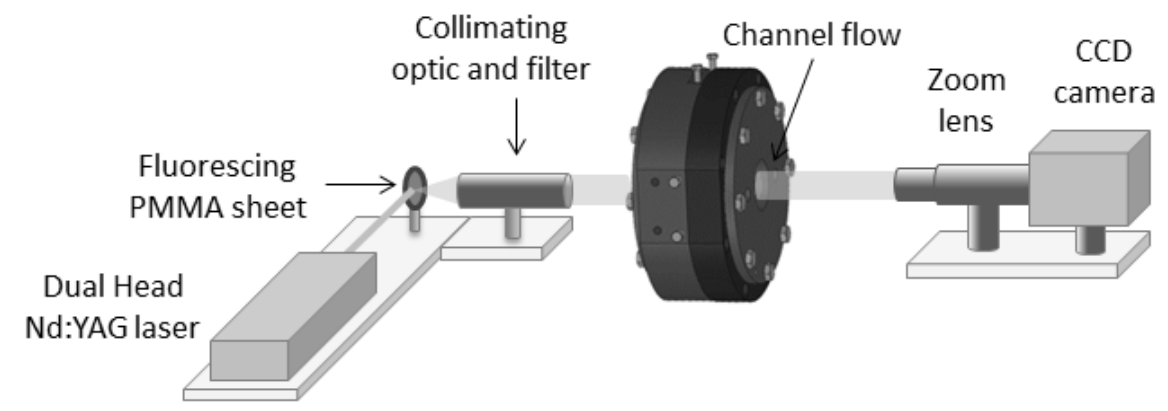

Figure 6: Shadowgraph-like optical set-up.
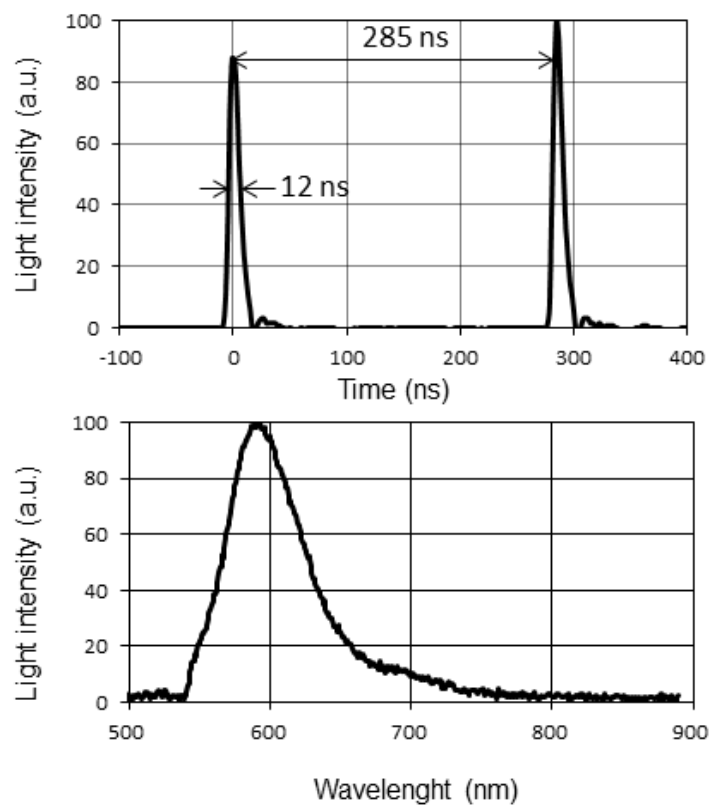

Figure 7: Light source temporal profile and spectrum measured by means of a fast photo-diode and a fiber based mini-spectrometer. 


\section{Shadowgraph-like images of the channel flow}

Fig. 8a displays an example of instantaneous image of the channel flow, recorded in the shadowgraph-like configuration previously described. In this raw image, the channel walls, the vapor cavities and the bubbles appear in black, when the liquid is essentially bright, as it should be in backlit imaging. However, grey structures are also visible in the image, as a result of a shadowgraph effect. Fig. 8 also displays a background image (Fig. 8b) and the computation of the quantity $\Delta I / I$ (Fig.8c), previously introduced in Eq.(7) and Eq.(8). I is the raw image intensity and $\Delta I$ is the relative change with respect to the undisturbed intensity, i.e. $\Delta I=I-I_{b}$ where $I_{b}$ is the background image intensity.

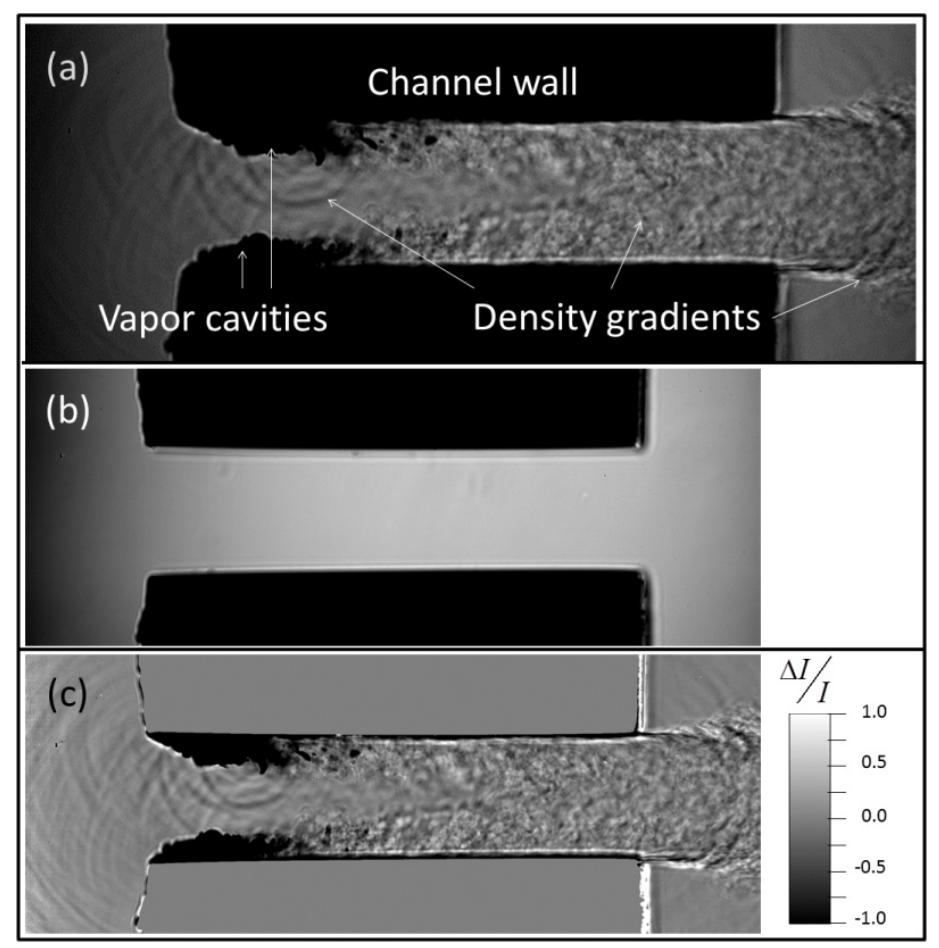

Figure 8 : Shadowgraph-like instantaneous image of the channel flow (a), background image (b) and normalized intensity $\Delta I / I$ (c).

Fig. 9 displays a sample of instantaneous shadowgraph-like images for increasing pressure drop from $\Delta P=4.5$ bar (a) to $\Delta P=40.2$ bar (j). The images of Fig.9 represent the quantity $\Delta I / I$ previously introduced. These images can be viewed as the results of a background subtraction and a normalization of the raw images. At low pressure drop, in Fig. $9 \mathrm{a}$ for $\Delta P=4.5$ bar, the flow is single phase and laminar. However, shadowgraph effects reveal density (refractive index) gradients 
as grey level variations. Density gradients are located in the shear layers at the channel inlet, in the out-going jet boundaries and along some streamlines of the flow. In a compressible fluid, density variation can be associated to pressure or temperature variation, or both together. In liquids, the temperature contribution generally overpasses the pressure contribution as a temperature drop of $1 \mathrm{~K}$ approximately corresponds to the same density variation than a pressure increase of $10 \mathrm{bar}$. In the present case, the origin of the density gradients is more ambiguous, as pressure gradients can become very large with increasing pressure drop. For $\Delta P=4.5$ bar (Fig. 9 a), the density gradients in the shear layers are probably associated to both a pressure gradient between the main flow and the recirculation zone, and a temperature gradient. The image also reveals some streamlines, which probably originate from a thermal marking of the flow, far upstream from the channel. This effect completely disappears at higher pressure drop (higher velocity) in the following images. At the channel outlet, we also suspect that both temperature and pressure gradient are involved. The temperature gradient would be due to viscous heating near the walls, in the boundary layer all along the channel. The pressure gradient would be associated to the pressure difference between the out-going jet and the downstream chamber.

For a larger pressure drop $\Delta P=15.8$ bar (Fig. 9 b), the density gradients in the shear layers appear more contrasted as the pressure difference between the recirculation zones and the main flow has increased. The gradients remain smooth and regular in the beginning of the shear layers (near the inlet corners) but they reveal developing instabilities at their end. Still in Fig. 9b, grey level random-like variations appear in the wake of the recirculation zones. These structures are developing from the walls to the center of the flow where they join together, about the middle of the channel. We interpret this structure as a signature of turbulence and associated density (pressure) fluctuations. 


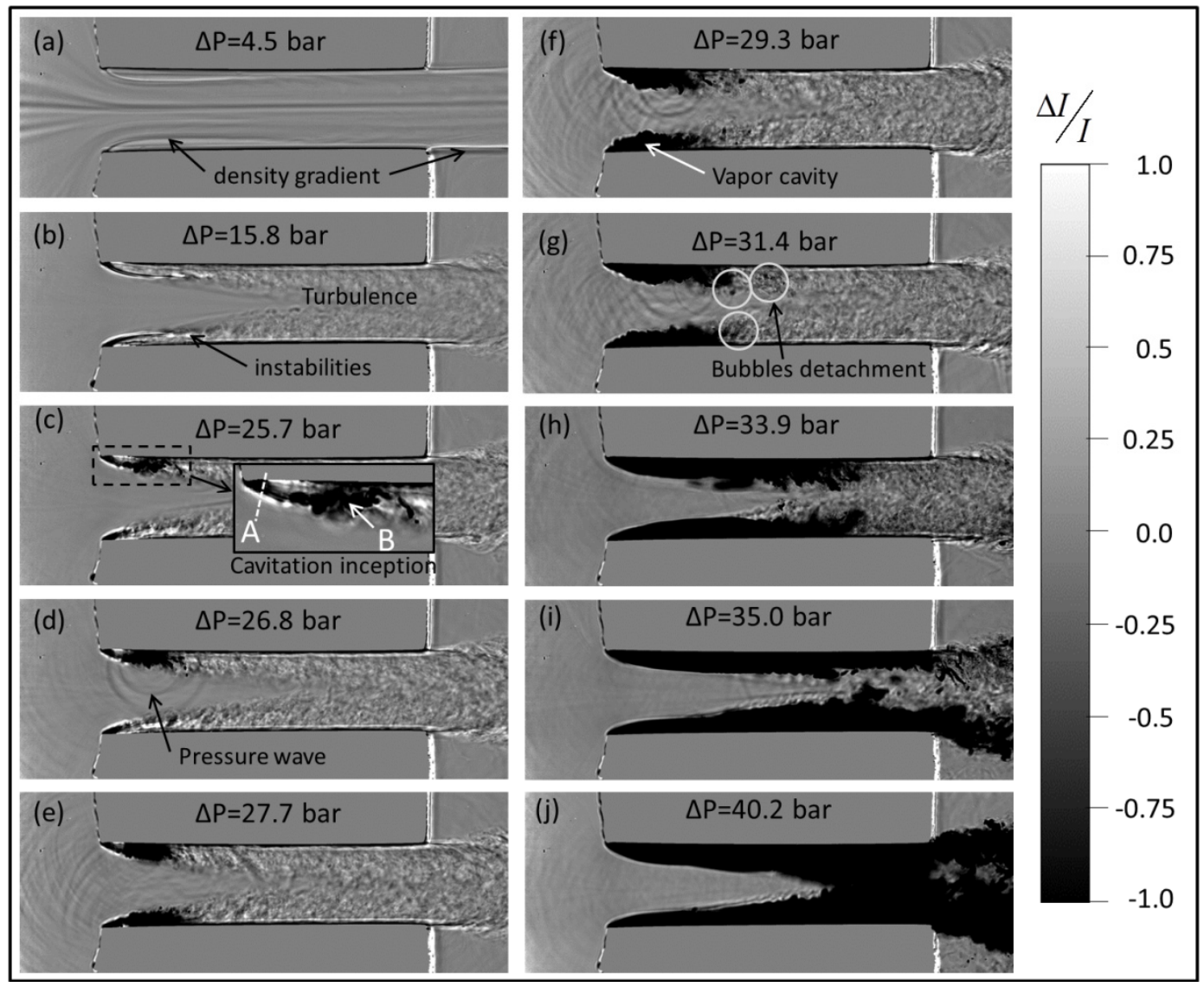

Figure 9: Sample of instantaneous shadowgraph-like images (after background subtraction and normalization) for increasing pressure drop from (a) with $\Delta \mathrm{P}=4.8$ bar to (j) with $\Delta \mathrm{P}=40.2$ bar. Upstream pressure is constant and equals 50 bar when downstream pressure is changed. The fuel flows from the left to the right.

Cavitation incepts for pressure drop $\Delta P$ of about 26 bar, in the upper part of the channel inlet, as shown in Fig. 9c and 9d. Shadowgraph-like images interpretation is not obvious because dark zones can be due to liquid-vapor interfaces or to density gradients. In Fig. 9c, it seems that cavitation incepts in the shear layer, relatively far from the inlet corner, where the lowest pressures are expected in average. Further in this paper, we will propose a scenario of cavitation inception in the shear layer, based on the results provided by the three optical techniques presented in this paper. In the lower shear layer of Fig. 9c, it seems that cavitation has not yet incepted. Here, the shear layer appears shortened with respect to the previous images as it seems that instabilities described in Fig. $9 \mathrm{~b}$ develop more intensively and sooner (closer to the channel inlet) for increasing pressure drop. This apparent asymmetry between upper and lower part of the channel is probably due to a weak asymmetry in the channel inlet geometry. 
For increasing pressure drop, the vapor cavities develop. They develop first in the upper shear layer (Fig. 9d), then in both upper and lower shear layers (Fig. 9e). From Fig. 9f-9i, vapor cavities fill the entire recirculation zones and progressively elongate, as the downstream pressure further decreases. The choked flow conditions previously shown in Fig. 4 are reached for $\Delta P=33.9$ bar (Fig. 9h). At this stage, vapor cavities have developed beyond the one-half of the channel length. For the largest pressure drop considered here $(\Delta P=40.2$ bar, Fig. $9 \mathrm{j})$, it seems that the vapor cavities join together and fill the entire channel section. In this extreme situation, cavities are probably no more 2D as it is hardly believable that all liquid transform into vapor.

Fig. 9 shows that shadowgraph-like images provide a wealth of information on the flow. In addition to the features previously described, one can see bubble detachments in the wake of vapor cavities (e.g. Fig. 9g). In Fig. 9d, two regular circles centered on the vapor cavity are clearly visible. We interpret these circles as pressure waves produced by bubble collapse. Several similar events are observed for larger pressure drop in Fig. 9e-9g. For even larger pressure drops, in Fig. 9h-9j, it seems that the amplitude of these pressure waves decreases and their occurrence becomes less frequent. Such pressure waves, produced by bubble collapse in an injector hole, have been considered numerically by Sezal et al (2009), but to our knowledge, no experimental observation of this phenomenon has been reported yet in similar configuration.

\section{Cavitation probablility}

The sample images of Fig. 9 have been selected to be representative of all the images recorded in the same condition, but some variations from one image to another can be observed. These variations are considered in Fig. 10 which displays images representing probabilities of cavitation occurrence. The same pressure drop conditions than for Fig. 9 are considered in Fig. 10. Cavitation probabilities have been built by adding 50 images recorded in the same conditions, after applying a threshold to each image in order to separate liquid from vapor. It is then assumed that dark pixels correspond to vapor and bright pixels to liquid. In other words, no distinction is made between vapor cavities and dark regions resulting from intense gradients. Excepted for the gradients in the shear layers, the other flow features appearing by shadowgraph effects in Fig. 9 
have been removed in Fig. 10 as a consequence of averaging. However, cavitation inception and cavities elongation are still clearly observed.

The Fig. 10 results offer some possibilities of quantification, taking the variability of the observations into account. For example, cavitation inception can be statistically associated to a critical pressure drop. An averaged cavitation probability has been calculated, in the rectangular area displayed with dashed boundaries in Fig. 10c. This area has been chosen to contain the first inception of cavitation, as previously identified in Fig. 9c, and to avoid the shear layer itself and density gradients that must not be considered in this calculation. The average cavitation probability in this area has been plotted as a function of pressure drop in Fig. 11. Considering an arbitrary threshold of $10 \%$ in cavitation probability, one can establish from Fig. 11 that cavitation starts in the upper shear layer for a pressure drop of $25.7 \pm 0.3$ bar. A similar analysis in the lower shear layer leads to a critical pressure drop of $27.7 \pm 0.4$ bar.
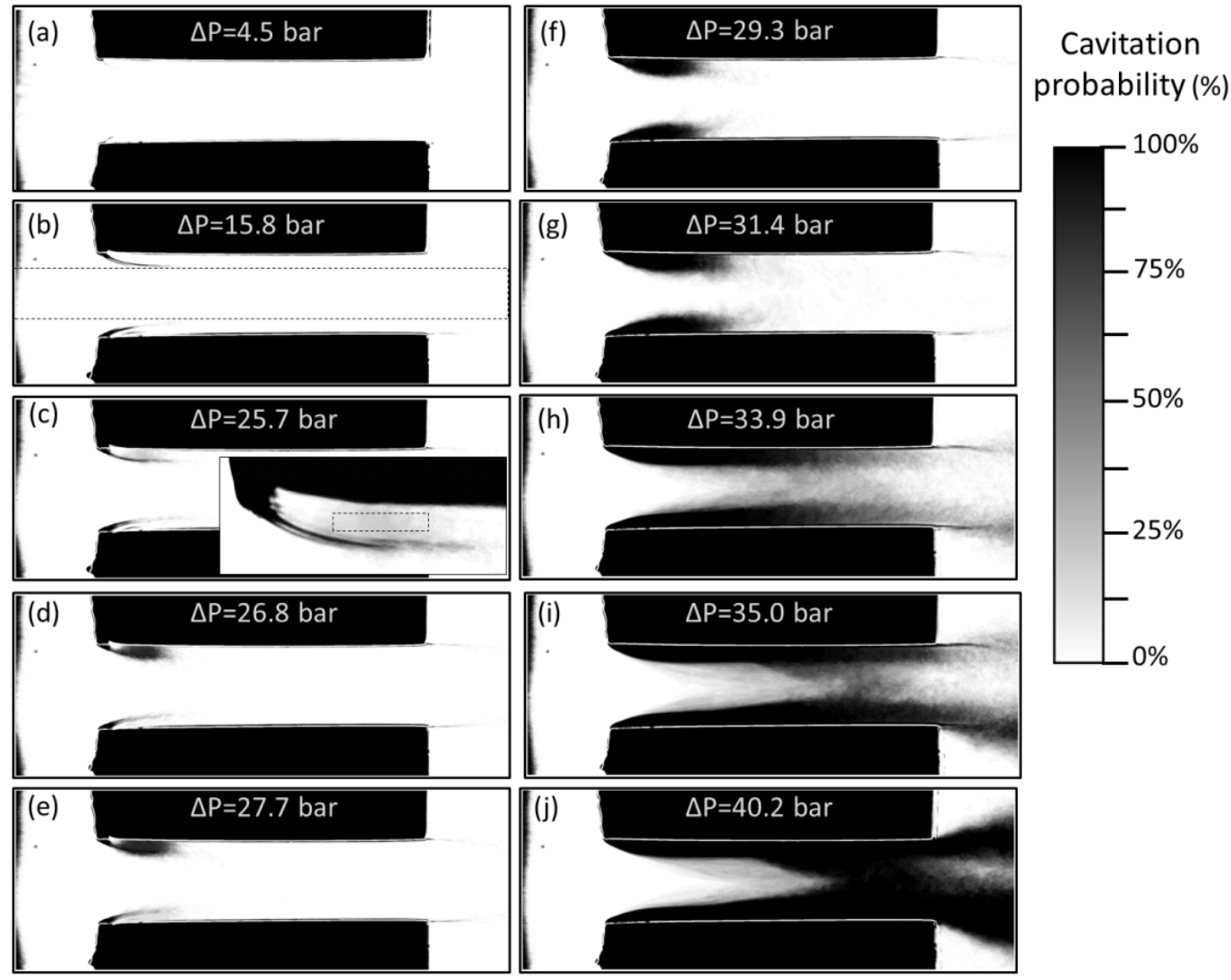

Figure 10: Cavitation probability deduced from shadowgraph-like images of the channel flow, for increasing pressure drop from (a) with $\Delta P=4.8$ bar to (j) with $\Delta P=40.2$ bar. Upstream pressure is constant and equals 50 bar when downstream pressure is changed. The fuel flows from the left to the right. 


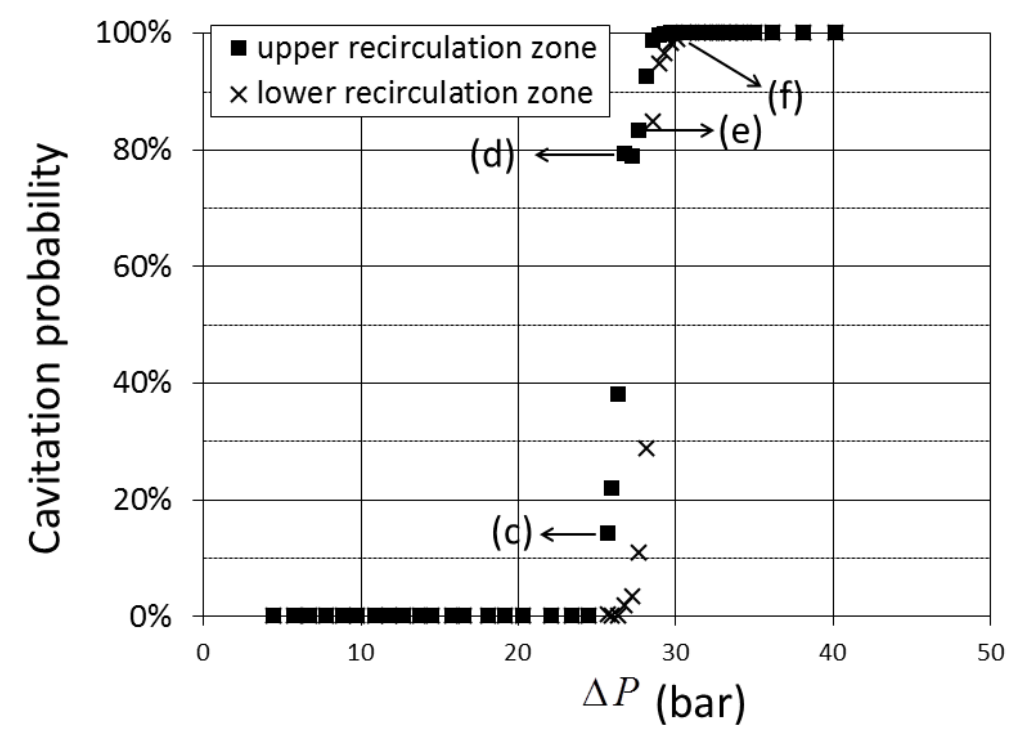

Figure 11: Averaged cavitation probability in recirculation zones. The letters (c) to (f) refer to figure 9 and 10 .

As shadowgraph-like images are sensitive to density gradients, they could be used, in principle, to measure the flow density. However, our attempts to reconstruct density fields have failed and there are several reasons for that. As previously mentioned, such a reconstruction requires a double integration. In addition, calibration targets would be necessary to obtain reliable quantitative information. Finally, and this is probably the main reason of our failure, Eq. (7) is not valid as the assumption of infinitesimal displacement is not satisfied. $\operatorname{Eq}(7)$ predicts that the shadowgraph effects are proportional to the distance $l$ and completely vanish for $l=0$. We then tried to adjust the distance $l$ (by displacing the channel by means of a linear translation stage) in order to reduce the shadowgraph effects. We found that these effects were still visible, with contrast in the same order of magnitude, whatever the value of $l$. It is then clear that Eq.(7) is not satisfied. The shadowgraph-like technique presented here cannot be used to obtain quantitative information on density fields. It is limited to provide qualitative information on density gradients.

\section{Schlieren imaging method}

The Schlieren method is an alternative technique to standard shadowgraph to measure density gradients. It is, in principle, sensitive to the first space derivative of the density along one direction. 


\section{Principle of the Schlieren method}

Fig. 12 is a schematic of a Schlieren imaging set-up. A point source is collimated to illuminate the test section $D$. A first lens, generally called Schlieren head, is used to generate a Fourier plane of the test section. A knife is placed in the Fourier plane and a second lens is used to image the test section on a CCD sensor. The technique differs from shadowgraph which requires a defocusing. In the Fourier plane, the knife edge cuts off a part of the point source image, which is located on the optical axis, in absence of perturbation (see (a) in Fig. 12). In the presence of refractive index (density) gradients in the test section, optical rays are deflected. Depending on the gradient sign, the light source image associated to the ray is moved upward or downward with respect to the undisturbed case (see (b) in Fig. 12), leading to a lower or a greater attenuation of light. Density gradients then appear darker or brighter on images. Assuming that rays undergo only infinitesimal deviations in the test section, neglecting geometrical aberrations of the lenses and considering a rectangular point source image, the relative change of intensity with respect to the undisturbed case can be written as (Merzkirch, 1974)

$$
\frac{\Delta I}{I} \approx \frac{f}{a} \int_{s_{0}}^{s_{1}} \frac{1}{n} \frac{\partial n}{\partial x_{i}} d s
$$

where $f$ is the focal length of the Schlieren head and $a$ is the width of the point source image in direction perpendicular to the knife edge. In Eq. (9), $x_{i}$ stands for $x$ in case of a horizontal knife edge (as considered in Fig. 12) and it stands for $y$ in case of a vertical knife edge. Assuming a 2D flow and still assuming infinitesimal ray displacements in $D$, Eq. (9) becomes

$$
\frac{\Delta I(x, y)}{I(x, y)} \approx \frac{f L}{a} \frac{1}{n(x, y)} \frac{\partial n(x, y)}{\partial x_{i}}
$$

The Schlieren method is then sensitive to the first derivative of the refractive index, perpendicularly to the knife edge. Note that under the assuming assumptions, it is not sensitive to ray displacement (shadowgraph effect), because images are focused onto the test section. In our case however, based on our experience with our shadowgraph-like configuration, sensitivity to the second derivative is expected in both directions, and parallel to the knife edge in particular. In the following, Eq. (10) will be however integrated to obtain 
qualitative information on the density fields that we expect to be more reliable, or at least complementary, with shadowgraph-like results.
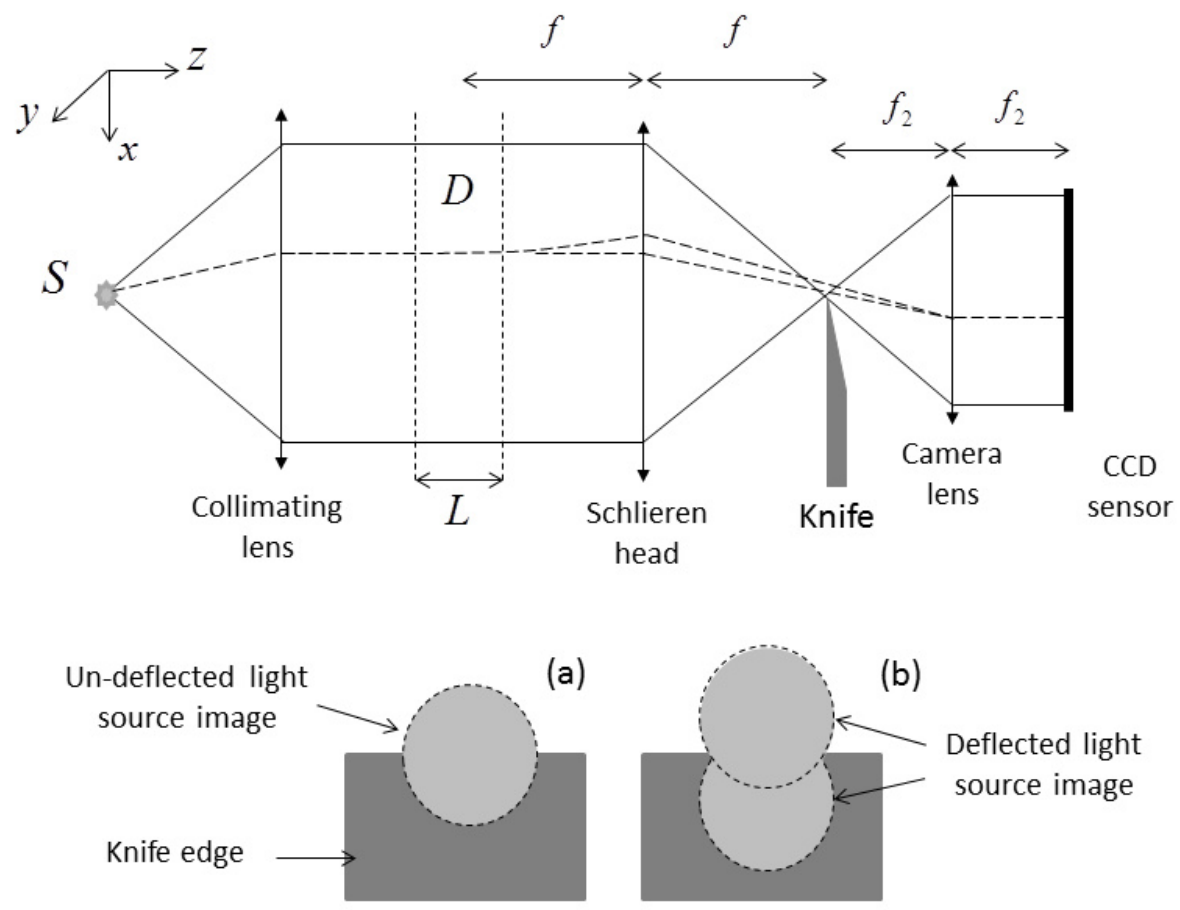

Figure 12: Schematic of Schlieren imaging method.

\section{Schlieren method with a vertical knife edge}

The knife edge is first placed vertically to obtain density information along horizontal direction ( $y$ coordinate). Fig. 13 displays some examples of Schlieren images $(\Delta I / I)$, for pressure drop equal to 15.7 bar (without cavitation, Fig. 13a) and 29 bar (with vapor cavities, Fig. 13c). Fig. 13b and 13d display intensity profiles along the center line of the channel, extracted from Fig. 13a and 13c respectively. These profiles have been averaged over 30 adjacent lines to limit the effect of noise. The remaining fast oscillations visible on the profiles are due to the turbulent structures and not to noise. The dashed lines plotted in these figures result from the integration (midpoint method) of the intensity profiles. These curves are similar to density profiles if we assume Eq. (10) as an approximation. The integrated profiles are very sensitive to any shift of intensity profiles, i.e. to the zero intensity definition. As a consequence, integration has been done under constraint, by forcing the density evolution to be null in the zone (A) delimited by the vertical dashed lines in the figure, near the channel exit. Note that this assumption is not exact as the pressure would decrease slowly in this region. We 
however assume that this decrease is negligible compared to the total pressure decrease in the channel. Further in this paper, the density fields obtained from interferometric imaging will confirm this assumption, with pressure variations lower than 1 bar in this specific region.

We estimate that the density profiles deduced from Schlieren images are not quantitatively reliable because the validity of Eq. (10) and underlying assumptions are questionable, because integrations are done under constraint and because no calibration has been done. We however assume that integrated profiles provide the general features of the flow density evolution along the channel centerline. These density evolutions will be helpful in the next section to interpret interferometric images and produce density fields of the channel flow.

The three techniques presented here are sensitive to density variations. It is not possible, in principle, to attribute this variation to a temperature change or a pressure change. However, one of the contributions can be sometime reasonably neglected compared to the other. In the present case, the density increase is about $6.510^{-2} \mathrm{~kg} \cdot \mathrm{m}^{-3}$ for 1 bar pressure increase, and about $-7.110^{-1} \mathrm{~kg} \cdot \mathrm{m}^{-3}$ for $1 \mathrm{~K}$ temperature decrease. A $1 \mathrm{~K}$ temperature decrease then produce the same effect on density than a 10.9 bar pressure increase. The global temperature elevation in the channel can be estimated following the work by Morini (2005), for a laminar flow with an adiabatic walls boundary condition. Temperature elevations of about $210^{-}$ ${ }^{2} \mathrm{~K}$ and $2.510^{-2} \mathrm{~K}$ are found for the conditions of Fig. 13a and Fig. 13b respectively. With Reynolds number in the order of $10^{4}$, the laminar flow assumption is not satisfied. However, assuming that the order of magnitude of these estimations is correct, the temperature effect on density would be equivalent to a pressure variation in the order of $0.2 \mathrm{bar}$, that is to say in the order of $1 \%$ of the pressure drop. The estimation of temperature increase given in (Morini, 2005) is global, that is to say, integrated over the whole channel section. One can suspect that the temperature profile in the channel section is not flat and that the temperature increase is greater in the boundary layers, near the walls, than in the centerline of the channel. Then, density variations deduced in Fig. 13 can be interpreted as pressure variations by neglecting temperature effect. Fig. 13 then provides the general feature of pressure evolution along the centerline of the channel. The pressure decreases at the channel inlet as the main flow section decreases. The pressure reaches a minimum value at the minimum flow section, 
caused by the flow separation. It then increases, as the flow section increases again, until a local maximum near about the flow reattachment. Beyond this point, the flow section remains constant and the pressure decreases slowly, until the end of the channel.

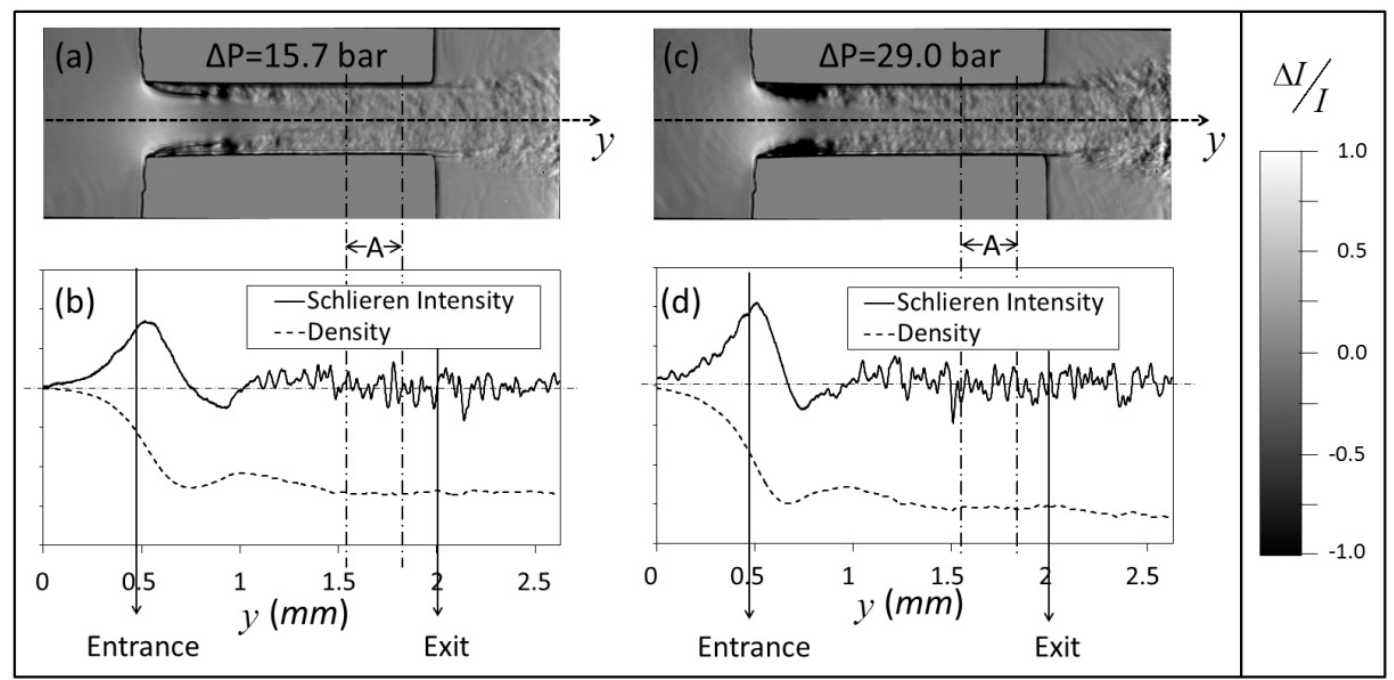

Figure 13: Examples of instantaneous images recorded using Schlieren method with vertical knife edge for $\Delta \mathrm{P}=15.8$ bar (a) and $\Delta \mathrm{P}=21$ bar (c). The curves under images represent intensity profiles along the centerline of the channel and density resulting from integration of these profiles.

\section{Schlieren method with a horizontal knife edge}

Using a horizontal knife edge, Schlieren method provides information on horizontal density gradient (along $x$ coordinate). Fig. 14 shows images obtained with a horizontal knife-edge for different pressure drops. Intensity profiles along the white dashed lines drawn on the images (averaged over 20 vertical lines) are shown together with the results of their integration with respect to coordinate $x$ (increasing from top to bottom). Curves plotted on the left side of Fig. 14 correspond to the intensity profiles, along a line crossing the shear layers in the separated flow region, near the channel inlet. These density profiles are obtained by integration under the constraint of equal densities at both end points, i.e. in upper and lower recirculation zones. Here again, this assumption is not completely satisfied as the effect of a weak asymmetry of the channel has been identified for cavitation inception (see Fig. 11 and associated comments in previous section). However, we also assume that the effect of this asymmetry on pressure fields can be neglected. This assumption will be confirmed in the next section, with the pressure fields deduced from interferometric images. In Fig. 14a, 
the flow symmetry at channel inlet is clearly visible in the image as the positive gradient in the upper shear layer appears dark and the negative gradient in the lower layer is bright. The integrated profile shows an increase of density from the recirculation zones to the center of the channel, with density gradient located in the shear layers. The density evolution along this profile is then essentially associated to a pressure evolution. In Fig. 14b and 14c, for greater pressure drops $\Delta P=15.7$ bar and $\Delta P=24.6$ bar respectively, the density profiles are similar but the pressure difference between the recirculation zones and the main flow increases. We note however that the apparent symmetry in images is broken. The lower shear layer remains essentially enlightened in Fig. 14b and Fig. 14c but it shows a dark line at its center. No equivalent bright line appears in the upper shear layer which remains completely dark. Such dark lines could be misinterpreted as vapor cavities but the pressure drop is much lower here $(\triangle P=15.7 \mathrm{bar}$ in Fig. 14b) than the pressure drop for which cavitation inception has been observed $(\Delta \mathrm{P}>25$ bar) in shadowgraph-like images (Figs. 9 and 10). This dark line is due to a reversing of the Schlieren intensity that will be discussed later in this section (limit angle of deviation in Schlieren technique). Fig. 14c, the integrated profile at channel inlet is strongly affected by this effect.

Intensity profiles in a cross-section of the out-going jet are also plotted on the right side of Fig. 14. The associated density profiles are obtained by integration under the constraint of equal densities at both end points, that is to says, assuming the same density above and below the jet in the downstream chamber. For $\Delta P=7.8$ bar, the density profile shows negative peaks at the jet boundaries. Such a density variation in the out-going jet can be explained as the consequence of both temperature and pressure evolution. The density slowly decreases with pressure form the jet center to the edges. At the jet boundaries, it undergoes a supplementary decrease associated to an increase of temperature due to flow heating near the walls, all along the channel. The temperature increase previously estimated for the whole channel section was very weak (in the order of $10^{-2} \mathrm{~K}$ ). From the Schlieren results, it seems that the temperature increase is essentially located near the channel walls, and in the wake these walls downstream the channel. The local temperature increase would be high enough to be visible on Schlieren images. An order of magnitude of these temperature increases will be provided further in the paper with density maps deduced from interferometric 
images. At the right side of Fig. $14 \mathrm{~b}$ and $14 \mathrm{c}$ (for pressure drops $\triangle P=15.7$ bar and $\Delta P=24.6$ bar respectively), the density profiles at the jet boundaries are dominated by the pressure decrease. However, an additional effect of temperature increase is likely to remain, as suggested by the aspect of the density profile on the right side of Fig. 14c, in the lower jet boundary.

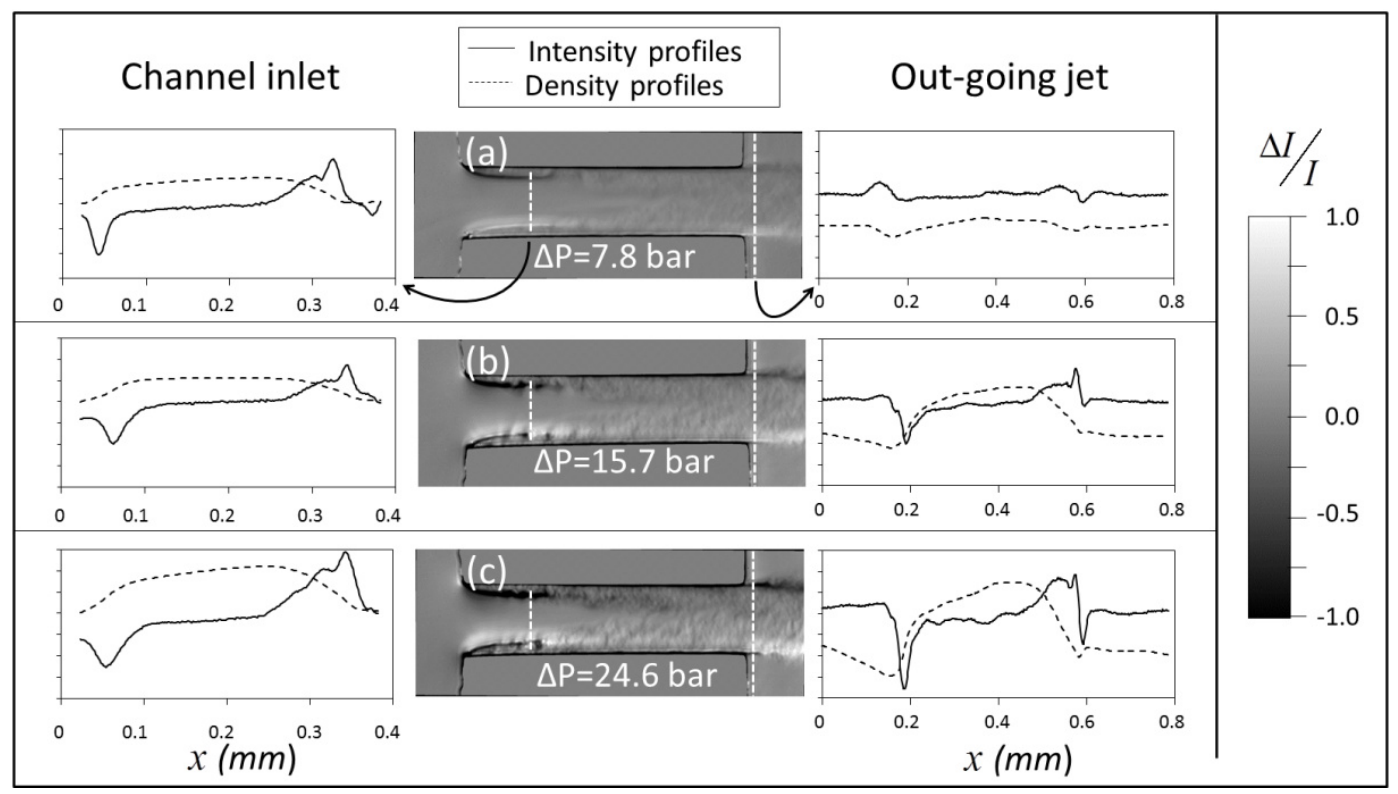

Figure 14: Examples of instantaneous images recorded using Schlieren method with horizontal knife edge for $\Delta P=7.8$ bar (a), $\Delta P=15.7$ bar (b) and $\Delta P=24.6$ bar (c). The curves on the left side represent intensity profiles in the separated flow region, near the channel inlet. The curves on the right side represent intensity profiles at the channel outlet in a cross-section crossing of the outgoing jet. The density profiles have been obtained by integration of the intensity profiles.

\section{Limit angle of deviation in Schlieren technique}

The dark lines observed in the lower shear layers in Fig. 14 are due to a limit angle of deviation in the Schlieren technique. As previously described in Fig. 12, density gradients induce optical ray deviations and displacements of the point source image in the Fourier plane. The stronger the gradient amplitude, the stronger the displacement and the recorded intensity (with appropriated gradient sign). However, as schematically shown in Fig. 15a, strong gradient may produce ray deviation $\varepsilon_{x}$ of the same amplitude than the collection angle $\alpha_{l}$ of the optic. Instead of increasing, intensity then decreases with increasing ray deviation. For ray deviation equal to the limit angle one can expect an intermediate intensity, equivalent to the undisturbed intensity (for zero deviation). Greater deviations then result in dark regions, where brightness is expected, as previously observed 
in Fig. 14b.

To underpin this interpretation, the density gradient and associated deviation angle can be estimated from the density maps to be introduced in the next section, and compared to the collection angle of the optical set-up. The dark line observed in the lower shear layer of Fig. 14b first appears for a lower pressure drop $\Delta P=9.8$ bar. In Fig. 15b, the beginning of the shear layer appears dark. Further from the inlet corner, where density gradients are smaller, the shear layer is still bright. In the middle of the shear layer, near about the dashed line of Fig. 15b, the Schlieren intensity is nearly equal to the undisturbed intensity. Then, the ray deviations at this point should be nearly equal to the collection angle of the Schlieren set-up. Fig. 15c shows the density map for approximately the same pressure drop (9.7 bar) than Fig. 15b (9.8 bar). From this map, the maximum pressure gradient along the dashed line is estimated to be between 1.5 and 2 bar. $\mu \mathrm{m}^{-1}$. The associated ray deviation angle can be easily estimated from Eq. (6) which provides the ray deviation $\varepsilon_{x}$ at the channel exit, in liquid. In air, after passing through the glass window, the deviation angle is nearly equal to $n \varepsilon_{x}$. Assuming a 2D flow, Eq. (6) then leads to

$$
n \varepsilon_{x} \approx L \frac{\partial n}{\partial x}
$$

Knowing the resolution of the Schlieren imaging system $(1.58 \mu \mathrm{m} / \mathrm{px})$, and the relation between refractive index and pressure, the estimated deviation angle ranges from $5.2^{\circ}$ to $6.9^{\circ}$. This estimation, perfectly agrees with the collection angle of the Schlieren imaging system, which is about $6^{\circ}$.

As well as for shadowgraph-like images, the interpretation of Schlieren images is not obvious. Dark zones are not systematically produced by the presence of vapor or by negative gradients but it can be due to an excessive positive density gradient. More generally, the Schlieren method provides general trends on the channel flow density but the reliability of the results is clearly questionable, especially for large density gradients. Another technique is then needed to obtain reliable and quantitative information on density fields. 

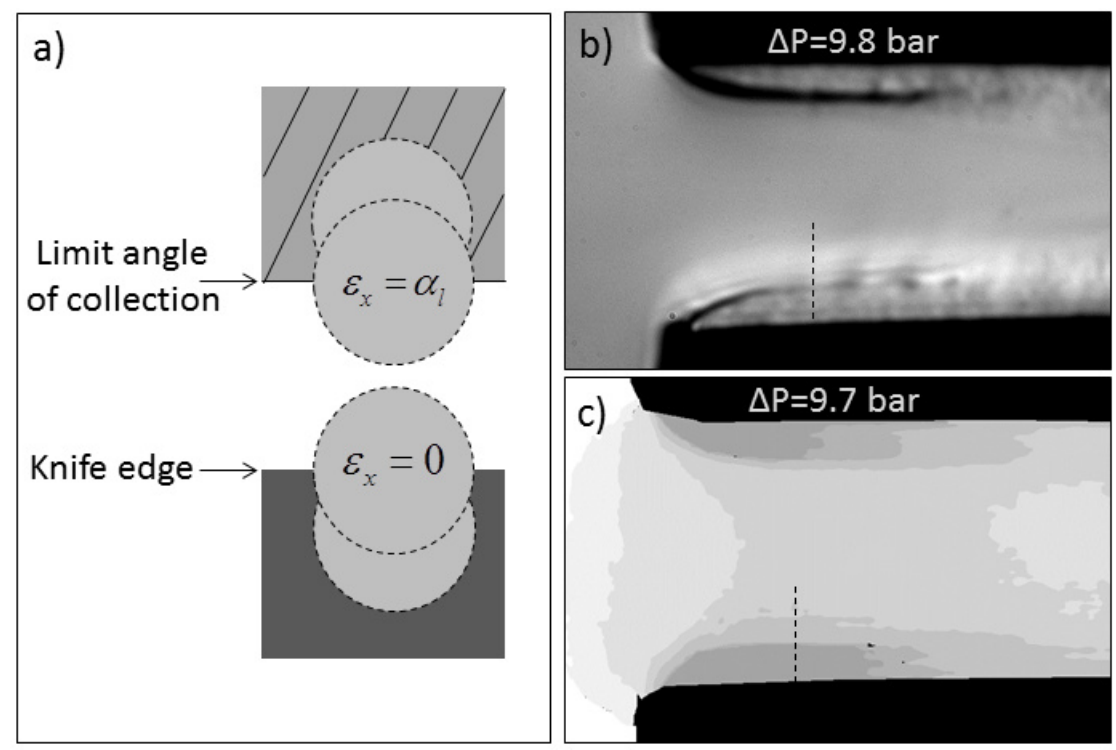

Figure 15: Schematic of the Schlieren intensity reversing due to limit angle of collection (a). Schlieren image with horizontal knife edge for $\Delta P=9.8$ bar (b) and pressure map deduced for interferometric imaging for pressure drop $\Delta P=9.7$ bar (c).

\section{Imaging interferometry}

Unlike the two imaging methods previously introduced, imaging interferometry is not sensitive to optical ray deviation. It is based on ray retardation (phase shift) measurement and it is then directly sensitive to density (or refractive index) rather than to its first or second derivative. Our interferometric arrangement is shown in Fig. 16. The green light $\left(\lambda_{\mathrm{Ar}}=514 \mathrm{~nm}\right)$ of a Argon-ion laser in continuous wave operation is used as the light source. The beam size is magnified by using a beam expander which consists of two convergent lenses. A pin-hole is placed in the focal plane of the first lens in order to achieve a spatial filtering of the beam. A beam splitter (BS) divides the beam into a reference beam and an imaging beam which illuminates the channel flow. The two beams are re-combined using a second beam splitter and they are transmitted through the camera lens. As a continuous illumination is used, the time resolution of the images is limited to the minimum exposure time of the camera. Interferometric images of the channel flow are then averaged over $20 \mu \mathrm{s}$. 


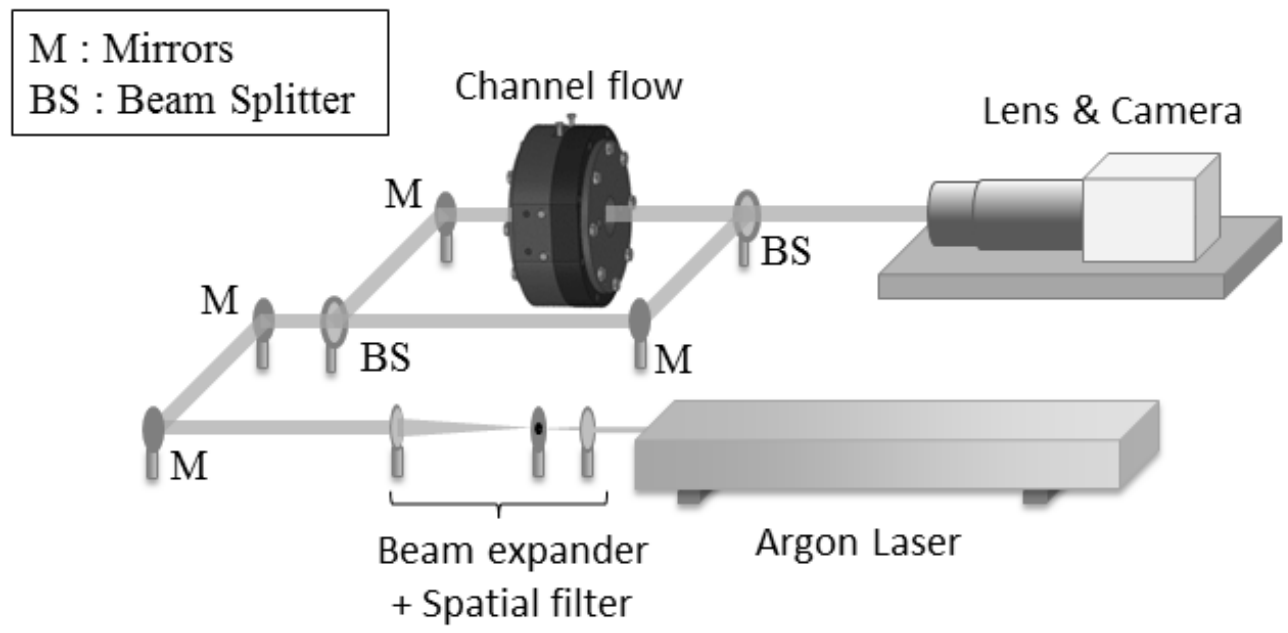

Figure 16: Interferometric imaging set-up.

Fig. 17 provides a sample of interferometric images of the channel flow, for pressure drops between $\Delta P=9.7$ bar and $\Delta P=35$ bar (Fig. 17a-17e). These images show a fringe pattern which depends on the density fields. Optical rays traveling through the channel flow are phase shifted, depending on the refractive index (or flow density). In association to the reference beam, these phase shifts lead to constructive or destructive interferences. The succession of bright and dark fringes in Fig. 17a-17e is then a signature of density evolution throughout the channel: at the channel inlet where pressure decreases fast, in the recirculation zones (for the lower pressure drops) and at the channel outlet. For increasing pressure drop, from Fig. 17a to Fig. 17e, the distance between fringes decreases throughout the channel, as density gradients increases in all parts of the flow. In interferometric images, channel walls appear in grey, as the reference beam contributes alone to images in these areas. Similarly, vapor cavities appear in grey in Fig. 17c to $17 \mathrm{e}$. 

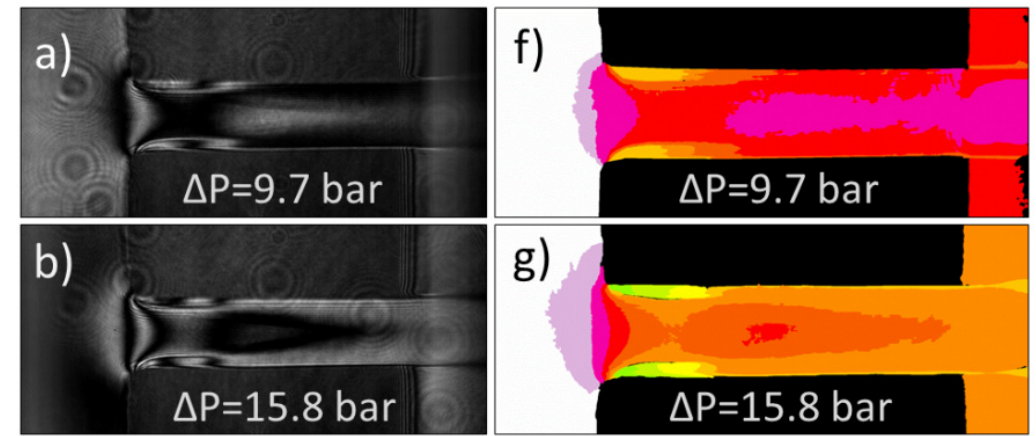

Pressure Density

(bar) (kg. $\left.\mathrm{m}^{-3}\right)$

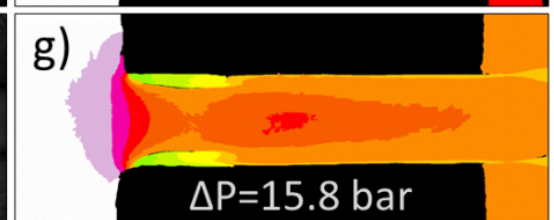

$50.0 \square 815.4$

\begin{tabular}{|l|l|}
47.0 & 815.2
\end{tabular}

$44.0 \quad 815.0$

$41.1 \quad 814.8$

$38.1 \quad 814.6$
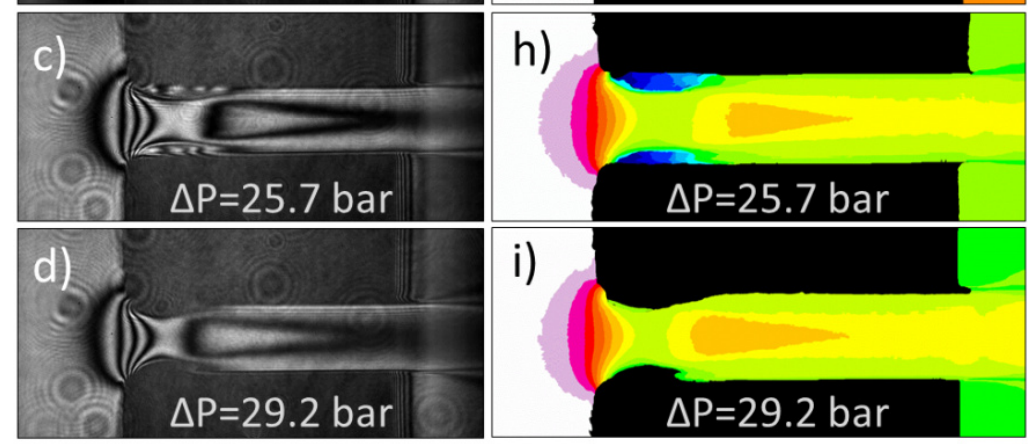

35.2

814.4

$32.2 \quad 814.2$

$29.3 \quad 814.0$

\begin{tabular}{l|l}
26.3 & 813.8
\end{tabular}

\begin{tabular}{l|l|l}
23.4 & 813.6
\end{tabular}

$20.5 \quad 813.4$

\begin{tabular}{l|l}
17.6 & 813.2
\end{tabular}

$14.7 \quad 813.0$

$11.8 \quad 812.8$

$8.9 \quad 812.6$

$6.0 \quad 812.4$
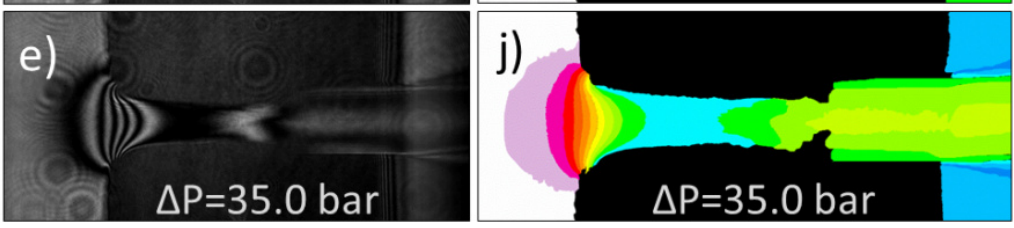

3.1

0.2

812.2

812.0

Figure 17: Interferometric images of the of channel flow (left) and density fields deduced from these images (right). A pressure scale, equivalent to the density scale, is proposed. It provides a more meaningful view of the results but it relies on a constant temperature assumption which is not satisfied everywhere.

To obtain quantitative information from imaging interferometry, we need a relation between optical ray phase shift and flow density. The optical path difference between a bright and a dark fringe is half the beam wavelength. The corresponding refractive index change is then

$$
\delta n=\frac{\lambda_{A r}}{2 L}
$$

To relate refractive index $n$ to flow density $\rho$, the following Lorentz-Lorenz relation is assumed

$$
\frac{n^{2}-1}{n^{2}+2}=k \rho
$$

where $k$ is a constant which depends only on material (the test oil in the present case). The constant $k$ is determined from known values of the refractive index $n_{0}=1.462$ and the density $\rho_{0}=820 \mathrm{~kg} \cdot \mathrm{m}^{-3}$ at standard pressure and temperature 
conditions $\left(P=1\right.$ bar and $\left.T=20^{\circ} \mathrm{C}\right)$. The refractive index has been measured by using a refractometer (NAR-1T). The density has been deduced from density measurement (Ndiaye et al, 2011) as a function of temperature in the range 10$130^{\circ} \mathrm{C}$ and pressure in the range 1-1400 bar. In the paper conditions (temperature $T=32^{\circ} \mathrm{C}$ and pressure $P$ ranging from 0 to $50 \mathrm{bar}$ ), the density increases almost linearly with refractive index. The refractive index change $\delta n$ between a bright and a dark fringe in the interferometric images is then associated to a density change $\delta \rho=2.010^{-1} \mathrm{~kg} \cdot \mathrm{m}^{-3}$. If a constant temperature assumption is assumed, the refractive index change $\delta n$ can be associated to a pressure change $\delta P$ which depends weakly on $P$ but is almost equal to 3 bar in the range $0-50$ bar.

In principle, density fields can be deduced from imaging interferometry, from a known density condition (upstream for example) and counting the number of fringes. However, such a construction is difficult in practice as the sign of the change is unknown. In other words, the density change between a dark and a bright fringe can be interpreted as an increase of $+\delta \rho$ or a decrease of $-\delta \rho$. The results obtained with the Schlieren method can be used to solve this indeterminacy and carefully reconstruct the density maps shown in Fig. 17f-17j. Density maps are presented with both a density scale bar and an equivelent pressure scale. The pressure scale is a direct translation of density in terms of pressure, under the assumption of constant temperature. This assumption is reasonable in the channel but not at channel exit where temperature effect are not always negligible. The density maps are built starting from the known upstream condition $\rho_{A}\left(P_{A}, T_{A}\right)$ and following the different paths shown in figure 18 , counting $\pm \delta \rho$ according to Schlieren results. Following path (1), the density profiles in the channel centerline previously shown in Fig. 13, are taken into account. The $2 \mathrm{D}$ consistency of the results is also checked by considering path (1') of Fig. 18 together with path (1). Through the out-going jet boundaries (end of path (1)), the Schlieren results shown in Fig. 14 are considered. For the lowest pressure drops $(\triangle P=9.7$ bar, Fig. $17 \mathrm{a}$ and $17 \mathrm{f})$, the density decrease due to viscous heating is taken into account. In this case, the back pressure is retrieved with deviation lower than $\delta P$ (one fringe). For the greater pressure drops, the temperature effect at jet boundaries was not clear in Schlieren results. However, the density at the jet boundaries must decrease under the downstream chamber 
density to retrieve the accurate back pressure. The density drop at the jet boundaries is in the order of $0.1 \mathrm{~kg} \cdot \mathrm{m}^{-3}$ (lower or equal to $\delta \rho=0.2 \mathrm{~kg} \cdot \mathrm{m}^{-3}$ ), corresponding to a temperature increases of about $0.15 \mathrm{~K}$. From these results, the temperature profiles in the outgoing jet are not flat, with a global temperature increase previously evaluated using (Morini, 2005) in the order of $10^{-2} \mathrm{~K}$ but a temperature increase, near the walls, ten times greater. In the recirculation zones, the density can be found following path (2) of Fig. 18, directly through the shear layers, but only for the lowest pressure drops (Fig. 17a and 17f). For greater pressure drops (Fig. 17b-17c), the density gradients in the shear layers become higher, the fringes get closer to each other and cannot be separated anymore. The path (3) of Fig. 18 is then used in these conditions to build the density field in the recirculation zone.

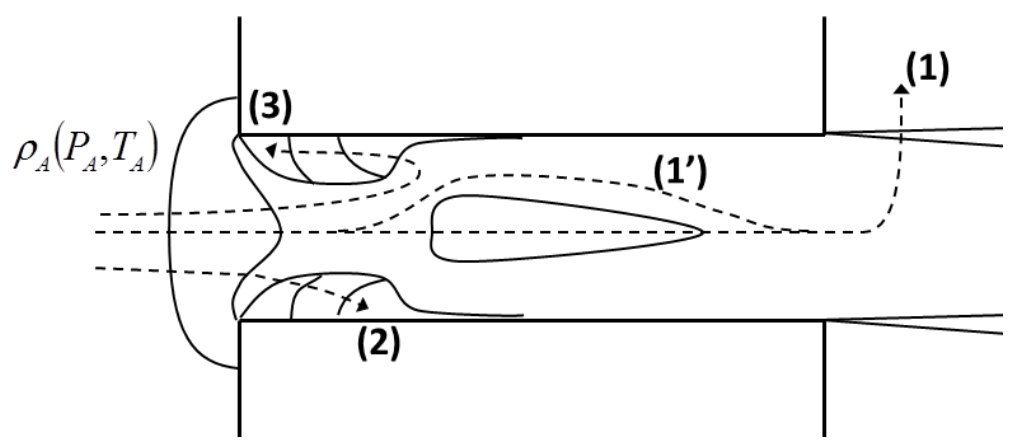

Figure 18: Schematic of the paths followed to build density field from interferometric images.

In Fig. $17 \mathrm{f}-17 \mathrm{j}$, the pressure evolutions along the channel centerline show the same features than the pressure curves deduced from Schlieren technique in Fig. 13. For all the pressure drop conditions, the pressure decreases rapidly at the channel inlet, it reaches a minimum, it increases again to reach a local maximum and then decreases slowly until the channel outlet. The minimum pressure point is located about the minimum section imposed by the flow contraction or the vapor cavities. It slowly moves toward the channel exit as the pressure drop increases from Fig. 17f to Fig. 17j. The minimum pressure value decreases with increasing pressure drop, as expected from the one-dimensional model introduced in section 3. However, even in choked flow condition (Fig. 17j), this pressure remains much greater than the vapor pressure. 


\section{Discussion}

In the previous section, three different optical techniques have been presented. The results have been partly discussed by considering the techniques one by one. In this section, we will further discuss our observations, based on comparisons between the three techniques results.

\section{Cavitation inception}

In the pressure field shown in Fig. $17 \mathrm{~h}$, for $\Delta P=25.7$ bar, the lower pressure

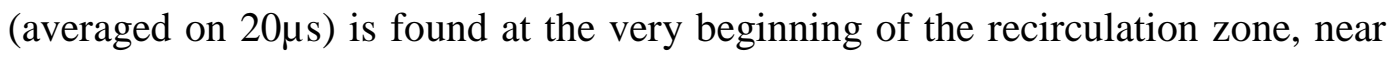
the inlet corner. One could expect that cavitation first incepts at this location, when pressure drops down to the fuel vapor pressure (to about zero). However, in the shadowgraph-like image of Fig. 9c, cavitation seems to first incepts in the upper shear layer, relatively far from the inlet corner. Here, the surrounded average pressure is higher. It ranges between 5 and 10 bar in the recirculation zone, just above the shear layer and it is about 25 bar, in the main flow, under the shear layer.

The detection of cavitation inception in the shear layer is based on distinction between density gradient and bubbles or vapor cavity in shadowgraph-like images. In these images, intensity variations produced by density gradients are roughly proportional to the second derivative of the density. As a consequence, in shadowgraph images, dark zones are generally associated to bright zones, as illustrated in Fig. 19 which provides examples of density profile and corresponding first and second derivatives. Fig. 9c, the right side of the upper shear layer (A, near the inlet corner) can be interpreted as an increase of density from the recirculation zone to the main flow, as the intensity distribution is similar to the scheme 1c of Fig. 19. In addition, the intensity profile at this location remains almost similar at this pressure drop condition $(\triangle P=25.7$ bar $)$ than at lower pressure drop in non-cavitating condition (Fig. $9 \mathrm{~b}$ for $\Delta P=15.8 \mathrm{bar}$ ). On the contrary, on the left side of the shear layer (B) in Fig. 9c, the cloud-like shape dark zone has no bright boundary and must be interpreted as a bubble cloud.

Note that the schematics of Fig. 19 can also be used to underpin some interpretation previously given in section 4 , as it provides a way to qualitatively distinguish simple increase of density from density peak. 


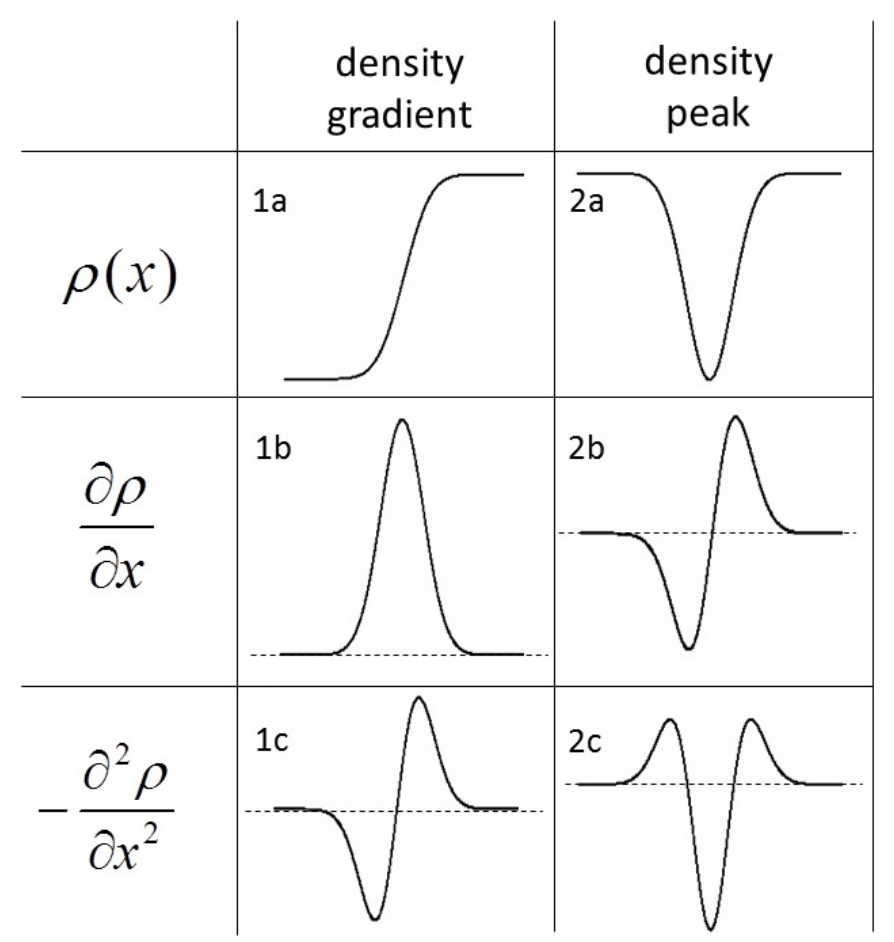

Figure 19: Schematic of simple density profiles and corresponding first and second derivatives.

\section{Pressure waves and bubble collapse}

Previously in this paper pressure waves have been observed in shadowgraph-like images of the channel flow. These pressure waves likely result from bubble collapse under the effect of an increase of pressure. These pressure waves are spherical and not quasi-2D, like most of the density gradients considered earlier in the paper. However, optical rays crossing the edge of the spherical waves, along a short chord, are actually deviated, resulting in a shadowgraph effect, as well as for the 2D gradients. Fig. 20 shows a couple of shadowgraph-like images, recorded with a time delay of $285 \mathrm{~ns}$. Several pressure waves can be observed in both images. However, these waves propagate too fast to enable the observation of the same wave in both images at this scale. On the contrary, the same detached vapor structure, probably composed of several bubbles, appears in both images, in the wake the main vapor cavity. Its displacement from Fig. 20a to Fig. 20b is about $16 \mu \mathrm{m}$ (14 pixels), leading to a velocity of about $56 \mathrm{~m} \cdot \mathrm{s}^{-1}$. The apparent volume of the detached structure clearly reduces from Fig. 20a to Fig. 20b, likely due to its partial collapse. In the same time, a pressure wave is visible in Fig. 20b. It is more contrasted than the others waves and it is centered on the vapor structure which has partially collapse, supporting our interpretation. 

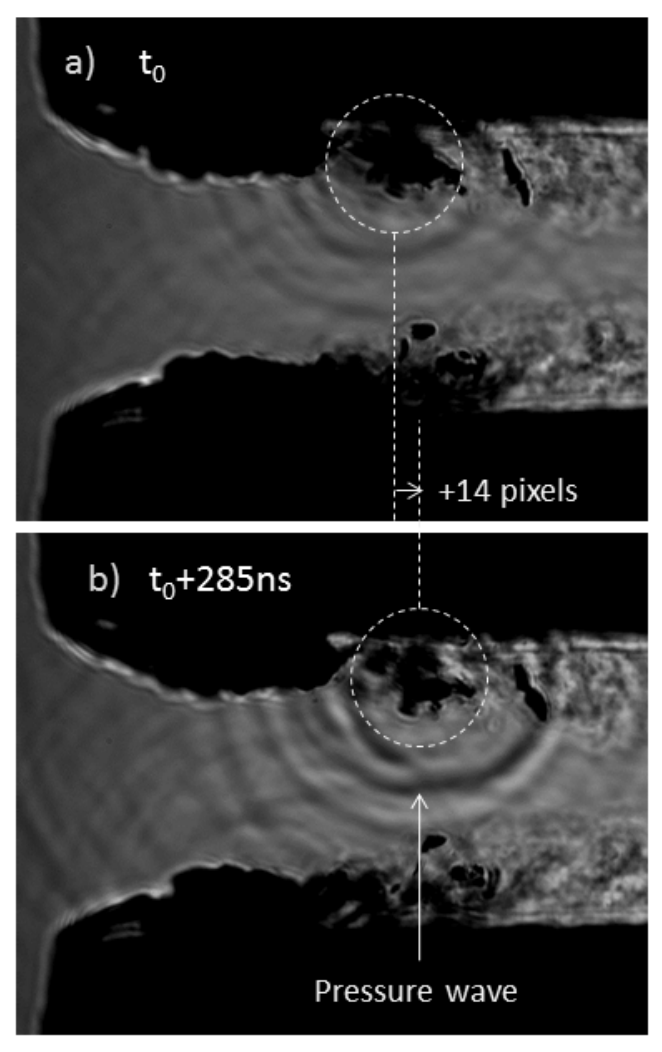

Figure 20: Apparent correlation between pressure waves and bubble collapse in the wake of vapor cavity.

As previously mentioned, pressure waves propagate at high velocity. It is then difficult to observe the same wave in two images, even with a very short time delay of $285 \mathrm{~ns}$. Most of the time, there are several pressure waves in the same image. It is then very difficult to associate a wave of the first image to the same wave, after propagation, in the second image. There is however a few examples (over several thousands of images) of such observation. One example is displayed in Fig.21. The pressure wave encircled in the first image (top of Fig. 21), has propagated during the $285 \mathrm{~ns}$ time delay between the two images. The distance traveled by the wave between the two images is $352 \mu \mathrm{m}$ upstream and $391 \mu \mathrm{m}$ downstream. The corresponding velocities are $1230 \mathrm{~m} . \mathrm{s}^{-1}$ and $1370 \mathrm{~m} . \mathrm{s}^{-1}$ respectively. Taking into account the flow velocity, which is $60 \mathrm{~m} . \mathrm{s}^{-1}$ (deduced from the mass flow rate measurement), the relative velocity of the wave is 1290 $\mathrm{m} . \mathrm{s}^{-1}$ in upstream direction, and $1310 \mathrm{~m} . \mathrm{s}^{-1}$ in downstream direction. These values are in the same order of magnitude than the sound speed measured in the test oil for the same temperature and pressure conditions ( $297 \mathrm{~K}$ and $20 \mathrm{bar}$ ), which is $\mathrm{c}=1335 \mathrm{~m} . \mathrm{s}^{-1}$. Note that during $12 \mathrm{~ns}$ (the duration of the pulsed illumination), the waves travel about $15 \mu \mathrm{m}$. The pressure waves are then not completely frozen in the images, leading to supplementary difficulties in trying to quantify them with 
the different techniques presented in this paper. Note also that no pressure wave has been observed for pressure drop $\Delta P$ lower than 25.7 bar, in absence of vapor bubble in the channel. This observation is another evidence of the cavitation inception at this pressure drop.

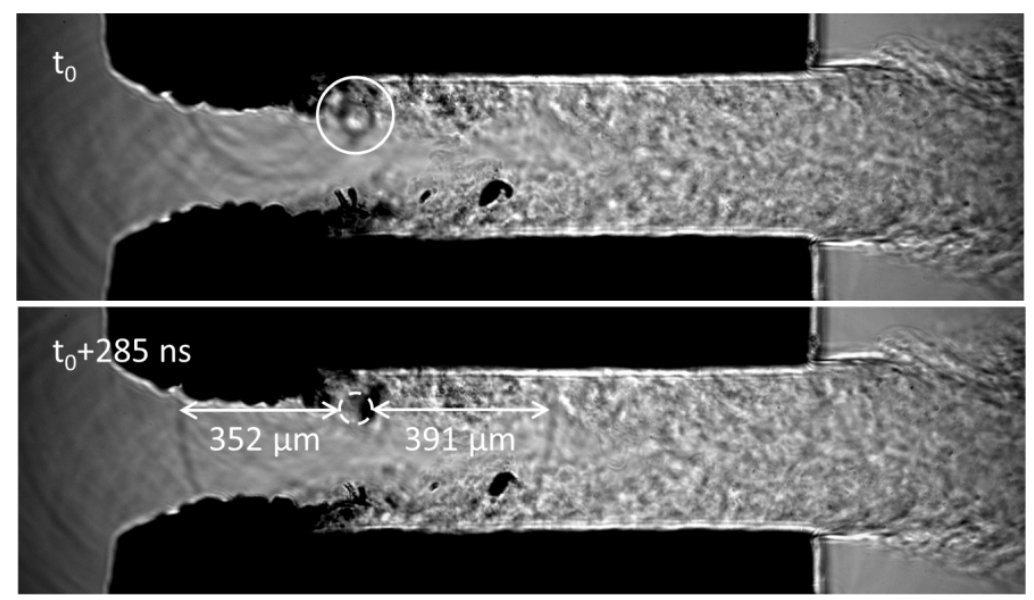

Figure 21: Couple of shadowgraph-like images (raw images) recorded with a time delay of $285 \mathrm{~ns}$. The pressure wave encircled in the first image (top) is still visible in the second image, after propagation. The fluid temperature is equal to $20^{\circ} \mathrm{C}$ and not $32^{\circ} \mathrm{C}$ as for the rest of paper.

\section{Bubble detachment}

In shadowgraph-like images, bubbles have been observed in the wake of vapor cavities. As previously discussed, bubbles likely collapse when they move to a zone of higher pressure, leading to a pressure wave occurrence. However, some bubble detachments are observed in high pressure zones. Fig. 9f for example, vapor bubbles are observed in the wake of the vapor cavity for $\Delta P=29.3 \mathrm{bar}$. At this location, for nearly the same pressure drop $(\triangle P=29.2 \mathrm{bar})$, the interferometric results of Fig. 17i show an average pressure of 26 bar. Let us consider the bubble in the rightmost circle of Fig. 9g. The bubble has travelled about $230 \mu \mathrm{m}$ from the vapor cavity. Its diameter is in the order of $20 \mu \mathrm{m}$. With a flow velocity in the order of $70 \mathrm{~m} \cdot \mathrm{s}^{-1}$, the life time of this bubble is found to be greater than $3 \mu$ s. Assuming a perfectly spherical bubble, the total collapse time can be approximated as a function of the initial radius $R_{0}$ of the bubble and the pressure $P$ in the surrounding liquid by (Rayleigh, 1917): 


$$
\tau \approx 0.915 \times R_{0} \sqrt{\frac{\rho}{P-P_{v a p}}}
$$

For a radius $R_{0} \approx 10 \mu \mathrm{m}$ and a pressure $\mathrm{P}=26 \mathrm{bar}$, the collapse time $\tau$ is equal to about 160 ns. Even with an initial bubble radius equal to the full cavity height, i.e. $R_{0} \approx 35 \mu \mathrm{m}$, the life time would remain lower than $600 \mathrm{~ns}$. The deviation between the Rayleigh model and the observed collapse time is then clear. It could be attributed to the bubble non sphericity. However, one can suspect that non spherical bubble with very large aspect ratio would be needed to explain such a large deviation. Another explanation would consist in assuming pressure fluctuations, relatively infrequent but high enough, to allow bubble survival over a long time. Such fluctuations could be generated by turbulence. There magnitude should be in the same order as the average pressure measured in the wake of the vapor cavity, i.e. in the order of 20 bar.

\section{Instabilities and cavitation inception in shear layers}

Section 4, instabilities have been identified in shadowgraph-like images, in the shear layers, at channel inlet. These instabilities are also visible in Schlieren images, especially when the knife edge is placed vertically as shown in Fig. 22. Instabilities develop with increasing pressure drop and produce vortices in the shear layer. In the Schlieren images, horizontal pressure gradients associated to these vortices appear as a succession of dark and bright spots, in the shear layer, relatively far from the inlet corner. For the lowest pressure drop, these intensity profiles are similar to the scheme $2 \mathrm{~b}$ of Fig. 19, corresponding to negative pressure peaks, similar to the scheme $2 \mathrm{a}$ of the same figure, at the vortices center. For the lowest pressure drop, the pressure difference associated to vortices can be roughly estimated. To do so, the Schlieren intensity profiles of Fig. 13 are compared to the profiles obtain from the pressure maps of Fig. 17. A pseudocalibration of the Schlieren images is then obtained and the pressure difference associated to vortices can be estimated to be about 1.5 bar in Fig. 22a (for $\Delta P=7.8$ bar) and to be about 2 bar in Fig. $22 \mathrm{~b}$ (for $\Delta P=9.8$ bar). For increasing pressure drop (Figs. 22c-22j), the dark spots become larger and closer to the channel inlet, as the pressure drop in vortices is amplified. Unfortunately, any estimation of these pressure drops becomes hazardous, for several reasons. 
First, dark spots are rapidly saturated (from Fig. 22c) as their intensity reaches zero (camera reading noise). For the largest pressure drops, the bright peaks intensity reversed as the deviation angle reaches the limit imposed by the collection optic, as previously detailed in case of horizontal knife edge. Next, Schlieren method is supposed to be sensitive to horizontal density gradients (with the vertical knife edge adopted here) but a shadowgraph effect along the perpendicular direction likely distorts the analysis. This effect is not negligible in the shear layers, where vertical pressure gradients increase with increasing pressure drop. Finally, we think that the 2D flow assumption is questionable in this particular location, especially for the largest pressure drops, just before cavitation inception.

A quantification of the density fields based on Schlieren images is hazardous but it is obvious from Fig. 22 that vortices are amplified with increasing pressure drop. Starting from an estimation of about 2 bar for the lowest pressure drop, one can reasonably presume that pressure variation associated to vortices may reach 5 or 10 bar for the greatest pressure drops considered in Fig. 22. In other words, we think that the pressure variations associated to vortices in the shear layer are in the same order of magnitude that the average pressure measured in the recirculation zone, just above this shear layer. The absolute pressure may then decrease under the critical cavitation pressure (let say vapor pressure) under the effect of strong instabilities. This scenario is compatible with our previous observations on shadowgraph-like images, where cavitation incepts in the shear layer, relatively far from the inlet corner. 

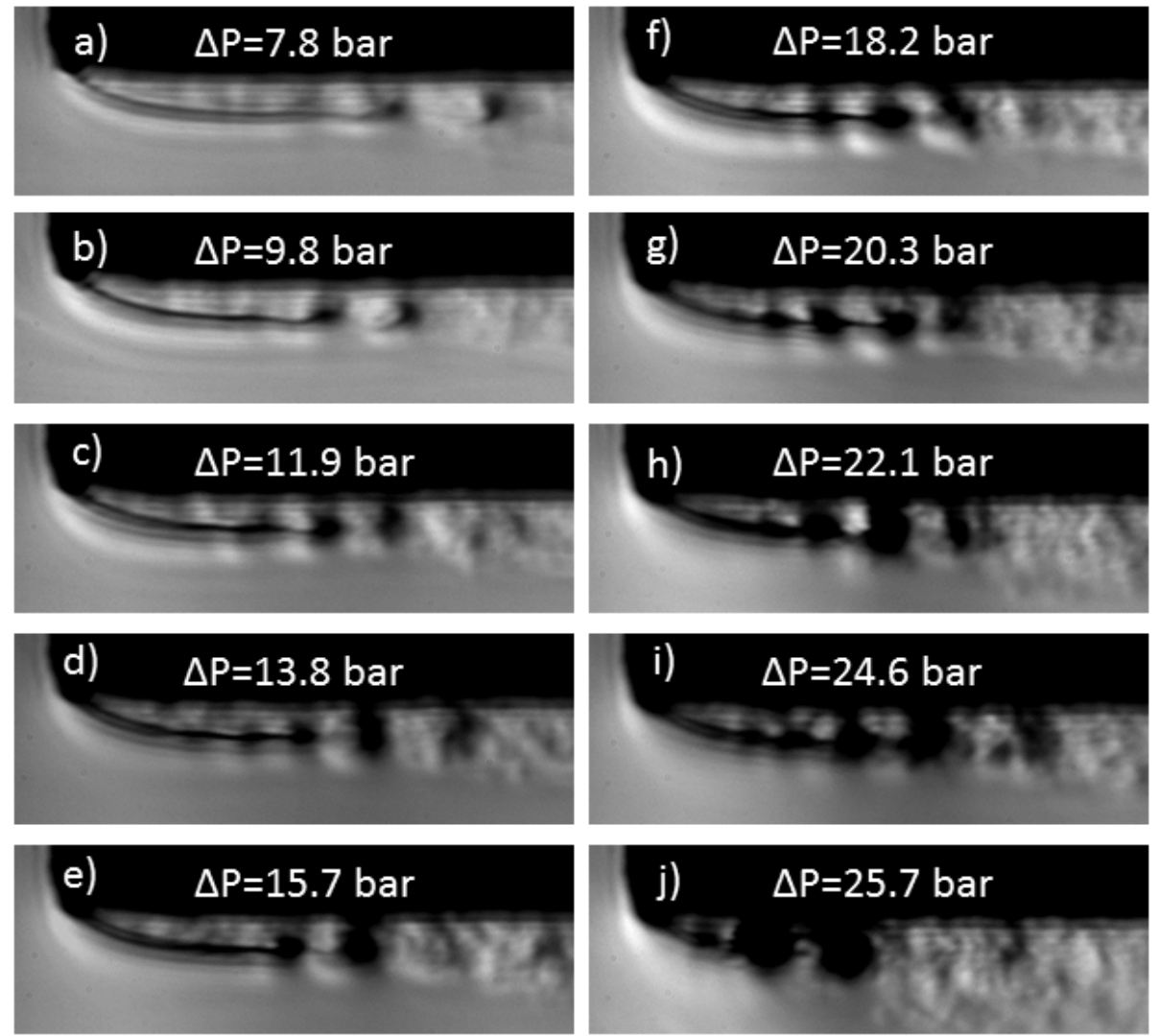

Figure 22: Growing instabilities in the upper shear layer visualized by Schlieren technique with vertical knife edge.

\section{Conclusion}

Visualizations of a quasi-2D channel flow have been carried out as a function the pressure drop by using three different optical techniques. Shadowgraph-like imaging provides information on the presence of vapor cavities and bubbles in the channel. It also provides qualitative information on density gradients, leading to difficulties in image interpretation. A Schlieren technique has been used to provide more reliable information on density gradients. For relatively low pressure drops, this technique has provided the general features of the density field along the center line of the channel, at channel inlet and at the out-going jet boundaries. However, the technique does not allow density field reconstruction when the channel flow shows higher density gradients. Then, an interferometric imaging technique has been used to quantify the density fields. Interfereometric images of the channel flow have been analyzed to reconstruct density fields, by taking advantage of the Schlieren results. Based on the three techniques results, the main features of the channel flow have been described. Cavitation incepts in 
the shear layers delimiting the recirculation zone and the main flow, at the channel inlet. It does not incept at the inlet corner, where the average pressure is minimum but a little further in the shear layer. Based on our observations we have proposed a scenario in which cavitation incepts under the joint effect of decreasing pressure in recirculation zones and instabilities developing in the shear layer. The paper results also suggest that the channel flow is highly turbulent and compressible. Bubble detachments have been observed. These bubbles likely collapse when they move through flow regions of higher pressure, leading to pressure wave occurrences. However, bubble detachments have been also observed in flow region where the average pressure is greater than 25 bar, suggesting the existence of pressure fluctuations in the same order of magnitude, generated by turbulence and carrying the bubbles.

\section{Acknowlegments}

This work takes place in the French collaborative program NADIA-bio (New Advance Diesel Injection Diagnosis for bio fuels). This program is supported by the French Automotive Cluster Mov'eo and funded by the DGCIS (Direction Générale de la Compétitivité, de l'Industrie et des Services), the Région Haute Normandie and the Conseil Général des Yvelines.

\section{References}

Badock C., Wirth R., Fath A., Leipertz A. (1999) Investigation of cavitation in real size diesel injection nozzles, Int. J. Heat and Fluid Flow 20:538-544.

Bergwerk W. (1959) Flow pattern in diesel nozzle spray holes, Proceedings of the Institution of Mechanical Engineers 173:655-660.

Chang J C, Huang S. B. and Lin C. M., (2006) Effect of inlet surface roughness, texture,and nozzle material on cavitation, Atomization and sprays 16:299-317.

Jung D., Wang W. L., Knafl A., Jacobs T. J., Hu S. J., and Assanis D. N. (2008) Experimental investigation of abrasive flow machining effects on injector nozzle geometries, engine performance, and emissions in a DI diesel engine, Int. J. Automotive Technology 9:9-15.

Merzkirch W. (1974) Flow Visualization, Academic Press, New York.

Morini G. L. (2005) Viscous heating in liquid flows in micro-channels, Int. J. Heat and Mass Transfer 48:3637-3647.

Ndiaye E. H. I, Bazile J. P. Nasri D. Boned C. and Daridon J. L. (2011) Private communication. (Laboratoire des Fluides Complexes et leurs Réservoirs, UMR CNRS 5150, Université de Pau).

Nurick W. H. (1976) Orifice cavitation and its effects on spray mixing, Journal of Fluids Engineering, 98:681-687. 
Payri R., Garcia J. M., Salvador F. J, Gimeno J. (2005) Using spray momentum flux measurements to understand the influence of diesel nozzle geometry on spray characteristics, Fuel $84: 551-561$.

Roosen P. (2007) Investigation of the Transient Behaviour of Cavitation Effects in Liquid Injection Nozzles. In : Transient Phenomena in Multiphase and Multicomponent Systems, WileyVCH Verlag GmbH \& Co. KGaA, pp 72-83.

Schmidt D. P., Rutland C. J., Corradini M. L. (1997) A Numerical Study of Cavitating Flow Through Various Nozzle Shapes, SAE Transactions, Journal of Engines 106:1644-1673.

Sezal I. H., Schmidt S. J., Schnerr G. H., Thalhamer M. and Förster M. (2009) Shock and wave dynamics in cavitating compressible liquid flows in injection nozzles, Shock Waves 19:49-58.

Soteriou, C., Andrews, R., and Smith, M., (1995) Direct Injection Diesel Sprays and the Effect of Cavitation and Hydraulic Flip on Atomization, SAE Technical Paper 950080. doi:10.4271/950080.

Winklhofer E., Kull E., Kelz E., Morozov A. (2001) Comprehensive hydraulic and flow field documentation in model throttle experiments under cavitation conditions, ILASS-Europe 2001, 26 sept, Zurich Switzerland,http://www.ilasseurope.org/ICLASS/ilass2001/ILASS2001.pdf.

Winklhofer E., Kelz E., Morozov A., (2003) Basic flow processes in high pressure fuel injection equipment, ICLASS 2003 july 13-17, Sorrento, Italy, http://www.ilasseurope.org/ICLASS/ iclass2003/fullpapers/1409.pdf. 